ÉGYPTE monde arabe

\section{Égypte/Monde arabe}

12-13 | 1993

Une économie en transition

\title{
Évaluation de la pauvreté en Égypte en fonction des données sur les ménages
}

Heba Al-Laithy et Hanaa Kheir al-Din

\section{(2) OpenEdition}

1 Journals

Édition électronique

URL : https://journals.openedition.org/ema/1257

DOI : $10.4000 /$ ema. 1257

ISSN : 2090-7273

Éditeur

CEDEJ - Centre d'études et de documentation économiques juridiques et sociales

Édition imprimée

Date de publication : 31 mars 1993

Pagination : 109-144

ISSN : 1110-5097

Référence électronique

Heba Al-Laithy et Hanaa Kheir al-Din, «Évaluation de la pauvreté en Égypte en fonction des données sur les ménages », Égypte/Monde arabe [En ligne], 12-13| 1993, mis en ligne le 08 juillet 2008, consulté le 07 juillet 2022. URL : http://journals.openedition.org/ema/1257 ; DOI : https://doi.org/10.4000/ema. 1257

Ce document a été généré automatiquement le 7 juillet 2022.

Tous droits réservés 


\section{Évaluation de la pauvreté en Égypte en fonction des données sur les ménages}

Heba Al-Laithy et Hanaa Kheir al-Din

1 L'Égypte a connu à partir de 1974 une décennie de croissance économique accélérée qui, conjointement à une politique sociale efficace visant à promouvoir justice sociale et bien-être, a fait progresser les revenus et le niveau de vie. Toutefois, la seconde moitié des années 80 a été éprouvante pour l'économie égyptienne :

2 - la croissance économique réelle a baissé annuellement de $5 \%$ en moyenne au milieu des années 80 ;

3 - l'inflation s'est maintenue à un taux oscillant entre 20 et $30 \%$;

4 - l'accumulation des arriérés a grevé lourdement la balance des paiements malgré les efforts d'ajustement et l'allégement de la dette consenti en 1987 par les membres du Club de Paris et par d'autres créditeurs ;

5 - des déficits budgétaires annuels, dépassant $20 \%$ du PIB, ont freiné l'économie et bridé le progrès social ;

6 - le chômage, surtout parmi la main-d'œuvre qui arrive sur le marché du travail, a connu une hausse aiguë et les salaires réels ont chuté dans la plupart des secteurs, affectant ainsi le niveau de vie en général et aggravant la pauvreté des groupes les plus démunis.

7 Face à ces problèmes, le gouvernement a adopté, dès 1986, un certain nombre de mesures. Celles-ci sont tout d'abord restées partielles et n'ont pu aboutir à une stratégie susceptible d'élever la productivité, de réduire le fardeau de la dette ou de promouvoir la croissance : la prudence excessive du gouvernement et son souci du bien-être des Égyptiens - tout particulièrement des groupes à faible revenu - ont retardé une réforme globale. Cependant, les difficultés économiques s'étant accusées, le gouvernement a adopté une politique plus dynamique et plus audacieuse qui allait 
aboutir à un programme d'ajustement structurel concrétisé par l'accord stand-by signé en mai 1991 avec le FMI.

Il faut espérer que ces mesures contribueront, par leur interaction, à réduire les déficits internes et externes, à encourager l'épargne nationale et promouvoir la productivité pour redonner une impulsion à l'économie, créer des emplois et assurer la stabilité des prix. Plusieurs des mesures du programme d'ajustement structurel visent à réduire les déficits budgétaires, à faire monter sensiblement les prix appliqués par les entreprises publiques, à abaisser les subventions à la consommation, à bloquer les salaires nominaux, de même qu'à assurer la relève des travailleurs employés dans les entreprises du secteur public et à resserrer le budget des services publics... Ces mesures peuvent s'avérer éprouvantes pour une bonne partie de la population, notamment les couches les plus vulnérables.

Il s'agit, dans cette étude, d'évaluer la nature et les caractéristiques de la pauvreté en Égypte et d'établir une comparaison entre différentes régions, afin de fournir aux responsables des données fiables leur permettant d'identifier les catégories concernées et de prévoir des mesures susceptibles de réduire la pauvreté dans le cadre de l'ajustement structurel. Nous procéderons en trois étapes: après avoir tenté d'identifier les couches pauvres, nous effectuerons une évaluation de la pauvreté par région et de manière prospective, puis nous nous efforcerons de dégager les caractéristiques de la population concernée en ce qui concerne la structure de la consommation, les sources de revenus et les composantes démographiques.

\section{Identifier les couches pauvres}

Quelles sont les catégories pouvant répondre à cette définition? Qu'entend-on par " pauvreté »? On peut avoir recours, pour la définir, à plusieurs indicateurs de niveau de vie : la totalité des revenus ou des dépenses du ménage ; les revenus ou les dépenses par tête; la consommation alimentaire par tête; la part du budget familial alloué à l'alimentation... ainsi que plusieurs autres indicateurs ne pouvant être réduits à une mesure monétaire unique, tels que l'accès à l'enseignement, la qualité de l'enseignement, la disponibilité en eau potable, les facilités de logement, etc. Etant donné les difficultés que l'on peut rencontrer pour évaluer ces facteurs et calculer les revenus, notamment dans les pays en voie de développement, on retiendra comme indicateurs, tout au long de cette étude, les dépenses totales par tête.

11 La plupart des études ${ }^{1}$ sur la pauvreté traitent des mérites respectifs des mesures adoptées pour lutter contre la pauvreté. La nature de cette dernière est tributaire de l'époque, du lieu, de la culture. La pauvreté est donc un concept relatif : elle reflète la situation des individus et des ménages dont les revenus ne suffisent pas à leur assurer un niveau minimal de bien-être.

\section{Seuils de pauvreté}

12 Le "seuil de pauvreté absolue » correspond à un niveau minimal de dépenses ou de revenus déterminé en termes réels dans un temps et un lieu donnés. Il se fonde sur le coût d'un niveau minimal de consommation alimentaire qui assure une ration calorique permettant d'éviter la malnutrition, ainsi que sur le coût d'autres produits primaires non alimentaires mais satisfaisant des besoins fondamentaux. La spécification de ces 
biens non alimentaires diffère d'un pays à l'autre : c'est à cet égard que la notion de pauvreté est relative ${ }^{2}$.

13 Le calcul du seuil de pauvreté absolue suscite généralement des problèmes empiriques et conceptuels: il est arbitraire et ne tient pas compte de l'évolution de la consommation alimentaire des ménages. Ces considérations ont amené plusieurs analystes à adopter un «seuil de pauvreté relative » fixé par rapport au niveau moyen de dépenses ou de revenus, et donc variable avec celui-ci. On fixe par exemple le seuil de pauvreté aux deux tiers, ou au tiers des revenus moyens par personne. Le «niveau de pauvreté » est alors calculé comme le pourcentage de la population dont les dépenses ou revenus sont en deçà du "seuil de pauvreté ». Toutefois, la pauvreté calculée en fonction de ce critère ne correspond pas au nombre absolu de pauvres ou à leur pourcentage par rapport à l'ensemble de la population, mais à leur répartition en deçà de ce seuil: le nombre de pauvres n'est donc pas un indicateur adéquat de la pauvreté. Enfin, dans un but de comparaison dans le temps, le seuil de pauvreté doit être calculé à prix constants.

M. Ravallion ${ }^{3}$ suggère de combiner les concepts de seuils de pauvreté absolue et relative en déterminant des seuils multiples pour comparer les différents niveaux de pauvreté. Lorsque l'on veut comparer la situation à deux dates, on fixe deux seuils de pauvreté : l'un déterminé en fonction des indicateurs de niveau de vie relevés à chacune de ces deux dates, l'autre reflétant les variations intervenues dans l'ensemble des critères de niveau de vie.

\section{Indices de pauvreté}

Outre l'indication du seuil de pauvreté, il faut encore prendre en compte trois éléments fondamentaux :

1. la prévalence de la pauvreté mesurée en fonction du nombre, ou du pourcentage, de la population totale vivant en deçà du seuil de pauvreté ;

2. l'intensité de la pauvreté mesurant l'écart entre le niveau de revenu des pauvres et le seuil de pauvreté ;

3. le degré d'inégalité parmi les pauvres, de manière à ce que le transfert de revenus des plus démunis vers ceux qui le sont moins accroisse la pauvreté mesurée, et vice-versa.

16 Toute mesure de la pauvreté devrait refléter ces facteurs. Un indice satisfaisant à cet égard a été suggéré par Foster, Gréer et Thorbecke ${ }^{4}$. Il se présente comme suit :

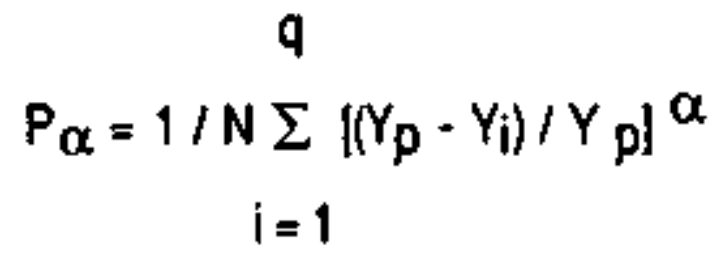

17 Yp indique le seuil de pauvreté, $\mathrm{Yj}$ les dépenses ou revenus de la i-ième personne pauvre, $\mathrm{N}$ l'effectif total de la population, q le nombre d'individus dont les dépenses ou les revenus sont en dessous du seuil de pauvreté, a étant un paramètre. Cet indice est basé sur la mesure de l'écart entre le seuil de pauvreté et les dépenses ou revenus de la personne pauvre, formulés comme fraction du seuil de pauvreté $(\mathrm{Yp}-\mathrm{Yj}) / \mathrm{Yp}$, le tout élevé à la puissance a puis sommé pour tous les ménages pauvres. L'indice tient compte non seulement de la prévalence et de l'intensité de la pauvreté, mais permet aussi de 
refléter le degré d'inégalité parmi les pauvres en faisant varier la valeur du paramètre $\alpha$. n'est pas facile à interpréter, comparé à $\mathrm{P}_{0}$ et $\mathrm{P}_{1}$, mais offre l'avantage de refléter le degré d'inégalité pour les pauvres ; autrement dit, plus l'inégalité de répartition entre les pauvres est forte, plus le degré global de pauvreté mesuré par $\mathrm{P}_{2}$ est élevé. Il faut relever enfin que cette classe d'indices de pauvreté est additive ; celui de la population totale est obtenu par la somme des indices des différents sous-groupes qui la composent.

\section{Mesure du seuil de pauvreté en Égypte}

Il n'existe en Égypte aucune enquête officielle spécifiquement destinée à évaluer la pauvreté, à analyser les caractéristiques des ménages pauvres ou à mesurer la disparité des revenus. Les seules données détaillées sont fournies par les enquêtes sur les 
budgets des ménages et les recensements de la population. À défaut d'une base de données plus appropriée, notre analyse reposera sur les résultats des enquêtes sur les budgets des ménages de 1974-1975 et de 1981-1982, sur les résultats préliminaires de l'enquête sur les revenus et dépenses, effectuée en 1990-1991, et sur le recensement de la population de 1986.

Certaines évaluations des seuils de pauvreté ont été effectuées en Égypte, chacune limitée par la méthodologie choisie et les données disponibles. K. Korayem ${ }^{6}$ a calculé le seuil de pauvreté en définissant le revenu minimal qui permet à un ménage d'acheter la quantité de produits alimentaires couvrant ses besoins biologiques, et de s'assurer un niveau minimal de biens et de services couvrant ses besoins non matériels. Ses évaluations se fondent sur un mode de répartition des revenus correspondant à l'enquête sur le budget des ménages de 1981-1982. S. Ibrahim ${ }^{7}$ et la Banque mondiale ont également calculé les seuils de pauvreté en Égypte, le premier en se fondant sur l'enquête sur le budget des ménages de 1974-1975, la seconde sur une mise à jour du travail du premier. Les deux évaluations sont inférieures à celles de K. Korayem.

Notre propre analyse est basée sur l'évaluation de deux seuils de pauvreté relative, un pour les régions rurales et l'autre pour les régions urbaines, ceci pour tenir compte des différences entre villes et campagnes ainsi que de l'autoconsommation dans les zones rurales. Le calcul fixe arbitrairement les seuils aux $2 / 3$ et $1 / 3$ de la dépense moyenne par tête dans chaque région pour les seuils de pauvreté et d'extrême pauvreté respectivement. Les résultats préliminaires de l'enquête sur les revenus et dépenses de 1990/1991 fournissent la répartition des ménages en fonction des dépenses par tête. Le tableau 1 donne la répartition par déciles des ménages rangés selon leur niveau de dépenses par tête. Ainsi, $10 \%$ des ménages de cet échantillon ont un niveau de dépenses par tête inférieur à 477,89 LE dans les régions urbaines et à 394,30 LE dans les régions rurales. $20 \%$ ont des dépenses par tête inférieures à 616,46 LE et à 467,83 LE dans les régions urbaines et rurales respectivement. $50 \%$ ont des dépenses par tête inférieures à 969,77 LE dans les régions urbaines et à 687,70 LE dans les régions rurales, etc.

Tableau 1 : Répartition des ménages (en déciles) 
selon leurs dépenses par tête (en LE), 1990/91

\begin{tabular}{|c|r|r|}
\hline n. de ménages (\%) & urbains & ruraux \\
\hline $10 \%$ & 477,884 & 394,299 \\
$20 \%$ & 616,462 & 467,818 \\
$30 \%$ & 725,707 & 538,657 \\
$40 \%$ & 841,160 & 610,367 \\
$50 \%$ & 969,771 & 687,696 \\
$60 \%$ & $1.030, .101$ & 765,026 \\
$70 \%$ & $1.241,311$ & 875,845 \\
$80 \%$ & $1.641,975$ & $1.024,160$ \\
$90 \%$ & $2.286,667$ & $1.310,365$ \\
\hline
\end{tabular}

Source : résultats préliminaires de l'enquête sur les revenus et dépenses 1991/91, CAPMAS, 1992

effectuées par K. Korayem ont été ajustées au niveau des prix de 1990/91, et ceci selon deux méthodes. La première a consisté à appliquer l'indice officiel global des prix à la consommation au seuil de la pauvreté qu'elle avait calculé, ce qui donne des estimations de 730,07 LE et 475,06 LE par tête, respectivement pour les ménages urbains et les ménages ruraux. La seconde méthode utilisée pour actualiser les estimations de K. Korayem ${ }^{8}$ a consisté à appliquer l'indice officiel des prix à la consommation de chaque composante des trois paniers alimentaires proposés par l'auteur comme devant assurer une ration calorique minimale, ceci afin d'évaluer le coût de ces paniers en 1990/91. D'après l'étude de l'auteur, le coût annuel de ces paniers était à l'époque de 490,99 LE et 333,65 LE par tête pour les régions urbaines et rurales respectivement. L'on a donc d'abord calculé la part des dépenses alimentaires dans les dépenses totales; pour les tranches de revenus concernées par l'enquête sur les budgets et la consommation des ménages de 1990/91, la totalité des dépenses par tête correspondant à la ration calorique minimale est de 733,32 LE pour les ménages urbains et de 436,90 LE pour les ménages ruraux. Les deux approches aboutissent à une évaluation du seuil de pauvreté plus élevée que la nôtre pour les régions urbaines, et moins élevée pour les régions rurales. Ces contradictions ne dépassent toutefois pas 1,5\% de notre évaluation pour les régions urbaines, et $10,1 \%$ pour les régions rurales. 


\section{Évaluation de la pauvreté par région et par période}

\section{Évaluation de la pauvreté par région}

30 Passons maintenant à une analyse détaillée de la pauvreté en fonction des seuils de pauvreté, évalués à $2 / 3$ et $1 / 3$ de la moyenne des dépenses par tête et par région et calculés en fonction des résultats préliminaires de l'enquête sur les revenus et dépenses de 1990-91. La classe $P_{\alpha}$ d'indices traités dans la section précédente a été calculée par zone urbaine et rurale, par région et par gouvernorat. L'impact des politiques diffère souvent selon les localités. Il faut donc distinguer les ménages ruraux des ménages urbains mais, même au-delà de cette distinction, il faut tenir compte d'un important facteur spatial, ces politiques affectant les marchés et dépendant de l'existence d'infrastructures. Il est par conséquent nécessaire, pour cerner l'impact de la politique régionale sur la pauvreté, de répartir les localités de manière plus fine et de ne pas se contenter d'opposer régions urbaines et régions rurales.

\section{a) Pauvreté dans les régions urbaines}

Les tableaux 2 et 3 fournissent les indices $\mathrm{P}_{\alpha}$ calculés d'après les deux seuils de pauvreté (722,56 LE et 361,28 LE respectivement) pour les différentes régions urbaines. Si l'on part de la ligne «toutes régions urbaines » (tableau 2), il apparaît que $\mathrm{P}_{0}$ - qui indique l'incidence de la pauvreté - atteint $29,15 \%$ des ménages : ce qui indique que pour 29,15\% des ménages urbains de l'échantillon, les dépenses par tête sont inférieures aux $2 / 3$ de la moyenne de l'ensemble de dépenses dans les régions urbaines; ces ménages doivent donc être considérés comme pauvres.

Tableau 2 : Indices de pauvreté des régions urbaines d'Égypte (seuil de pauvreté $=722,56$ LE)

\begin{tabular}{|c|c|c|c|c|c|c|c|}
\hline \multirow[t]{2}{*}{ Régions } & \multicolumn{3}{|c|}{ Indices de pauvreté. } & \multicolumn{3}{|c|}{ Contribution* } & \multirow{2}{*}{$\begin{array}{l}\% \mathrm{n} . \\
\text { ménages }\end{array}$} \\
\hline & Po & $P_{1}$ & $\mathrm{P}_{2}$ & Po & $P_{1}$ & $P_{2}$ & \\
\hline Le Caire & 19,53 & 4,75 & 1,64 & 19,12 & 18,03 & 17,26 & 27,97 \\
\hline Giza & 25,87 & 6,84 & 2,40 & 9,08 & 9,56 & 9,18 & 10,28 \\
\hline Alexandrie & 27,96 & 7,78 & 3,16 & 1,224 & 13,54 & 15,10 & 12,82 \\
\hline Clde Suez & 25,16 & 6,15 & 2,03 & 3,26 & 3,15 & 2,86 & 3,77 \\
\hline Bse -Egypte & 32,19 & 7,56 & 2,67 & 30,54 & 28,40 & 27,57 & 27,66 \\
\hline $\begin{array}{l}\text { Hte-Egypte } \\
\text { Ttes réoions }\end{array}$ & 45,49 & 12,11 & 4,55 & 25,49 & 26,88 & 27,70 & 16,33 \\
\hline urbaines"* & 29,15 & 7,36 & 2,68 & 100 & 100 & 100 & 100 \\
\hline
\end{tabular}

Source : estimations tirées des résultats préliminaires de l'enquête du CAPMAS, op. cit.

- Contribution de chaque région à lindice de pauvrelé = (indice de pauvrelé de la région) (\% nb. de ménages) / indice national de pauvrelé.

"Y compris les gouvernorats frontaliers. 

des cas, insignifiantes, à l'exception de celles qui apparaissent entre Le Caire, d'une part, et la Haute-Égypte et Alexandrie d'autre part, ainsi qu'entre la Haute-Égypte et les autres villes de Basse-Égypte (cf. tableau A3 de l'annexe - non reproduit; ndlr). En calculant la contribution de chaque région à la pauvreté urbaine au niveau national (tableau 2), il s'avère que la plus forte revient aux villes de Basse-Égypte, en raison d'un poids démographique relativement élevé et d'une pauvreté elle-même relativement élevée, mesurée par par $\mathrm{P}_{0}, \mathrm{P}_{1}$ et $\mathrm{P}_{2}$; elles sont suivies par les villes de Haute-Égypte, dont les indices de pauvreté sont relativement plus élevés mais qui comptent une population moins nombreuse, puis par Le Caire.

38 En ce qui concerne l'extrême pauvreté, il apparait que 4,65 \% des ménages des régions urbaines en sont frappés. La situation décrite ci-dessus reste valable pour l'extrême 
pauvreté en Haute-Égypte (ménages dont les dépenses par tête sont inférieures à 361,28 LE par an). Dans la plupart des régions, le phénomène est important, exception faite du Caire où l'indice de prévalence de l'extrême pauvreté est le moins élevé. Cependant, si l'on considère également les indices $\mathrm{P}_{1}$ et $\mathrm{P}_{2}$, le Caire vient en deuxième place après les régions urbaines de Haute-Égypte (tableau 3). L'incidence de la forte pauvreté est de $8,38 \%$ dans les régions urbaines de Haute-Égypte, mais seulement de 4,07 \% au Caire. Les valeurs de $\mathrm{P}_{1}$ sont respectivement de l'ordre de $2,73 \%$ et de $1,27 \%$ pour la HauteÉgypte et le Caire, et celles de $\mathrm{P}_{2}$ de $0,97 \%$ et de $0,54 \%$ pour les deux régions, un pourcentage plus élevé que dans toutes les autres régions.

Tableau 3 : Indices d'extrême pauvreté des régions urbaines d'Égypte (seuil de pauvreté $=361,28$ LE)

\begin{tabular}{|c|c|c|c|c|c|c|c|}
\hline \multirow[t]{2}{*}{ Régions } & \multicolumn{3}{|c|}{ Indices de pauvreté } & \multicolumn{3}{|c|}{ Contribution } & \multirow{2}{*}{$\begin{array}{l}\% \text { nb. } \\
\text { ménages }\end{array}$} \\
\hline & Po & $P_{1}$ & $\mathbf{P}_{2}$ & Po & $P_{1}$ & $P_{2}$ & \\
\hline Le Caire & 4,07 & 1,27 & 0,54 & 24,49 & 27,70 & 28,68 & 27,97 \\
\hline Giza & 3,23 & 0,70 & 0,24 & 7,14 & 5,62 & 4,63 & 10,28 \\
\hline Alexandrie & 5,56 & 1,21 & 0,51 & 15,31 & $12 ; 11$ & 12,36 & 12,82 \\
\hline Cl de Suez & 2,52 & 0,92 & 0,46 & 2,04 & 2,72 & 3,27 & 3,77 \\
\hline Bse-Egyple & 3,69 & 1,00 & 0,40 & 21,94 & 21,65 & 20,90 & 27,66 \\
\hline Hte-Egypte & 8,38 & 2,73 & 0,97 & 29,08 & 30,20 & 30,15 & 16,33 \\
\hline urbaines & 4,65 & 1,28 & 0,53 & 100 & 100 & 100 & 100 \\
\hline
\end{tabular}

excessivement pauvres (29\%) se trouve en Haute-Égypte (la part des zones urbaines de cette région, par rapport au nombre total de ménages couverts par l'échantillon, est environ de l'ordre de $16 \%$ ). Quant au pourcentage des ménages cairotes très pauvres au sein de l'ensemble de cette catégorie de ménages au niveau national, il dépasse la proportion qu'occupent les ménages cairotes dans l'échantillon national. Par conséquent, toute politique tendant à réduire la pauvreté doit tenir compte en particulier de la Haute-Égypte urbaine et de ceux qui vivent en dessous du seuil d'extrême pauvreté au Caire.

\section{b) Pauvreté dans les régions rurales}

42 Comme l'indique le tableau 4, la prévalence de la pauvreté est de 20,79\%, ce qui signifie que pour environ $21 \%$ des ménages de l'échantillon, les dépenses par tête sont inférieures à 485,87 LE par an (à savoir 2/3 des dépenses moyennes par tête dans les régions rurales). L'indice d'écart par rapport au seuil de pauvreté $\mathrm{P}_{1}$ est évalué à 3,91\%.

Égypte/Monde arabe, 12-13| 1993 
L'on peut évaluer cet écart à 0,0391 \% LE x 485,87 LE = 19 LE par an et par personne dans les régions rurales, ce qui représente environ $2,61 \%$ de la moyenne des dépenses par tête. L'indice $\mathrm{P}_{2}$ pour toutes les régions rurales est évalué à 1,23\%. Quel que soit l'indice $\left(\mathrm{P}_{0}, \mathrm{P}_{1}\right.$ ou $\left.\mathrm{P}_{2}\right)$ appliqué, le classement des différentes régions rurales ne varie pas. La Haute-Égypte est la plus pauvre, suivie par Giza et la Basse-Égypte (Isma'iliyya ne comptant pas de pauvres).

Tableau 4 : Indices de pauvreté des régions rurales d'Égypte (seuil de pauvreté $=485.87$ LE)

\begin{tabular}{|l|c|c|c|c|r|r|c|}
\hline \multirow{2}{*}{ Régions } & \multicolumn{3}{|c|}{ Indices de pauvreté } & \multicolumn{3}{c|}{ Contribution } & \% nb. \\
\cline { 2 - 7 } & $\mathrm{PO}_{\mathrm{O}}$ & $\mathrm{P}_{1}$ & $\mathrm{P}_{2}$ & \multicolumn{1}{c|}{$\mathrm{P}_{\mathrm{O}}$} & \multicolumn{1}{c|}{$\mathrm{P}_{1}$} & \multicolumn{1}{c|}{$\mathrm{P}_{2}$} & ménages \\
\hline Giza & 16,67 & 2,75 & 0,71 & 3,91 & 3,43 & 2,79 & 4,87 \\
Isma'iliyya & 0,00 & 0,00 & 0,00 & 0,00 & 0,00 & 0,00 & 0,98 \\
Bse-Egypte & 12,83 & 1,77 & 0,50 & 34,04 & 24,94 & 23,26 & 55,15 \\
Hte-Egypte & 33,12 & 7,56 & 2,50 & 58,96 & 71,63 & 74,95 & 37,00 \\
Tles régions & & & & & & & \\
rurales* & 20,79 & 3,91 & 1,23 & 100 & 100 & 100 & 100 \\
\hline
\end{tabular}

Source : enquête CAPMAS op. cit.

* Y compris les gouvernorats frontaliers.

43 Le test de signification appliqué à $\mathrm{P}_{0}$ révèle que les différences entre zones rurales sont significatives pour toutes les régions, excepté Giza et la Basse-Égypte (tableau A4 de l'annexe - non reproduit ; ndlr). Les différences entre les $\mathrm{P}_{1}$ de Basse-Égypte et des autres régions rurales sont également significatives, mais celles entre les $\mathrm{P}_{2}$ des diverses régions ne le sont pas, à une seule exception près (tableau A5 et A6 de l'annexe - non reproduit; $n d l r)$.

Dans l'ensemble de la pauvreté nationale, la part de la Haute-Égypte est la plus grande, suivie de la Basse-Égypte et de Giza, bien que la Basse-Égypte compte un plus grand nombre de ménages dans l'échantillon que Giza et la Haute-Égypte réunies.

Le tableau 5 montre que l'on compte, dans les régions rurales, 5,04\% de ménages extrêmement pauvres; il indique également que la plus forte concentration d'extrême pauvreté se trouve en Haute-Égypte (celle-ci contient, selon l'indice $\mathrm{P}_{0}, 60 \%$ des ménages très pauvres). 
Tableau 5 : Indices d'extrême pauvreté des réglons rurales d'Égypte (seuil de pauvreté = 242,93 LE)

\begin{tabular}{|l|r|r|r|r|r|r|c|}
\hline \multirow{2}{*}{ Régions } & \multicolumn{3}{|c|}{ Indices de pauvreté } & \multicolumn{3}{c|}{ Contribution } & \% nb. \\
& \multicolumn{1}{|c|}{$\mathrm{PO}_{\mathrm{O}}$} & $\mathrm{P}_{1}$ & $\mathrm{P}_{2}$ & $\mathrm{PO}_{\mathrm{O}}$ & $\mathrm{P}_{1}$ & $\mathrm{P}_{2}$ & \\
\hline & & & & & & & \\
Giza & 7,64 & 2,64 & 1,10 & 7,38 & 9,75 & 9,87 & 4,87 \\
Isma'lliyya & 0,00 & 0,00 & 0,00 & 0,00 & 0,00 & 0,00 & 0,98 \\
Bse-Egypte & 2,89 & 0,90 & 0,42 & 31,54 & 37,53 & 42,70 & 55,15 \\
Hte-Egypte & 8,14 & 1,88 & 0,70 & 59,73 & 52,72 & 47,42 & 37,00 \\
Ttes régions & & & & & & & \\
rurales* & 5,04 & 1,32 & 0,54 & 100 & 100 & 100 & 100 \\
\hline
\end{tabular}

Source : Enquête CAPMAS op. cit.

\section{c) Pauvreté par gouvernorat}

L'incidence et l'intensité de la pauvreté, ainsi que son inégalité de répartition, varient considérablement d'un gouvernorat à l'autre. Les tableaux A7 à A10 (voir annexe) montrent comment se répartissent la pauvreté et l'extrême pauvreté respectivement par régions urbaines et rurales dans les divers gouvernorats. Dans tous les gouvernorats de Haute-Égypte (tableau A7), les indices de pauvreté sont au-dessus du niveau national, exception faite de Giza et d'Assouan. Quel que soit l'indice appliqué, le gouvernorat le plus pauvre est celui du Fayoum. L'étendue de la pauvreté y est de l'ordre de $66,67 \%$; mesurée en fonction de $\mathrm{P}_{1}$, elle est près de trois fois plus élevée qu'au niveau national dans les régions urbaines, et environ dix fois plus élevée qu'à Isma'iliyya, où elle est la plus faible de tous les gouvernorats. Le degré de pauvreté urbaine au Fayoum est également le plus élevé de tous les gouvernorats (environ trois fois plus que la moyenne nationale). Le Fayoum contribue à concurrence de $4,05 \%$ à l'indice de pauvreté au niveau national, mesuré par $\mathrm{P}_{0}\left(5,18 \%\right.$ par $\mathrm{P}_{1}$ et $6,09 \%$ par $\left.\mathrm{P}_{2}\right)$, bien qu'il représente seulement $1,78 \%$ des ménages de l'échantillon. Autrement dit, la pauvreté y est particulièrement importante, non seulement eu égard au nombre de pauvres, mais aussi eu égard au degré de leur pauvreté. Sohag et Assiout suivent, où les coefficients $P_{1}$ d'écart par rapport au seuil de pauvreté sont les plus élevés parmi les autres gouvernorats. Menoufia vient au quatrième rang des gouvernorats les plus pauvres en fonction des indices $\mathrm{P}_{0}$ et $\mathrm{P}_{1}$, et au troisième rang en fonction de $\mathrm{P}_{2}$. L'incidence de la pauvreté à Menoufia atteint $48,54 \%$ d'après $\mathrm{P}_{0}$, soit environ 1,7 fois la moyenne nationale. Elle est à l'origine de $4,05 \%$ de la pauvreté nationale mesurée par $\mathrm{P}_{0}$, de $4,49 \%$ et $4,85 \%$ de la pauvreté nationale mesurée respectivement par $\mathrm{P}_{1}$ et $\mathrm{P}_{2}$, tandis que cette province ne représente que $2,45 \%$ de l'échantillon. Les indices de pauvreté de la province d'Isma'iliyya sont les plus faibles; Damiette et Matrouh suivent, cette dernière en raison de ses activités commerciales avec la Libye.

L'incidence de la pauvreté au Caire est de 19,52\%, tandis que l'intensité de la pauvreté, mesuré par $\mathrm{P}_{1}$, n'est que de $4,75 \%$. Mettant l'accent sur les ménages les plus pauvres, l'indice $\mathrm{P}_{2}$ souligne que la disparité au sein des ménages pauvres atteint 1,66 \% au Caire. Parmi les 22 gouvernorats recensés, Le Caire vient au 17e rang sur $P_{1}$ et $P_{2}$, et au 16e sur 
$\mathrm{P}_{1}$. Enfin, bien que le Caire forme $27,97 \%$ des ménages de l'échantillon, il ne contribue que pour $19,12 \%, 18,03 \%$ et $17,26 \%$ à la pauvreté saisie au niveau national, mesurée respectivement par $\mathrm{P}_{0}, \mathrm{P}_{1}$ et $\mathrm{P}_{2}$. Alexandrie vient au $13 \mathrm{e}$ et au $12 \mathrm{e}$ rang des indices $\mathrm{P}_{0}$ et $\mathrm{P}_{1}$, ce qui reflète un pourcentage relativement élevé de ménages pauvres dans cette ville. Les caractéristiques fondamentales révélées par le tableau A7 demeurent pour l'essentiel lorsque l'on considère l'extrême pauvreté. Le tableau A8 (annexe) indique que ce sont les régions rurales du Fayoum, de Sohag et d'Assiout qui en souffrent le plus. Leur contribution à l'extrême pauvreté au niveau national est parmi les plus élevées. Les gouvernorats d'Isma'iliyya et de Matrouh ne comptent pas de ménages urbains très pauvres.

Au niveau rural (tableaux 9 et 10 de l'annexe), ce sont encore les gouvernorats de Haute-Égypte (sauf Giza et Assouan) qui accusent les plus fortes incidences et intensité de la pauvreté, le Fayoum venant en tête sur Pi et $\mathrm{P}_{2}$, mais Minya tenant la première place sur Prj. S'agissant des populations rurales de Giza, de Qena et d'Assouan, le tourisme et la proximité du Caire (pour Giza) sont à l'origine d'un niveau de vie relativement plus élevé. Il n'y a pas de ménages pauvres dans les régions rurales d'Isma'iliyya, et Damiette a les indices de pauvreté les plus faibles. La répartition de l'extrême pauvreté rurale entre les divers gouvernorats (tableau A10 en annexe) correspond dans une très large mesure à la pauvreté mesurée par le tableau A9.

Tous les indicateurs soulignent le fait que la pauvreté et l'extrême pauvreté sont concentrées en Haute-Égypte (à l'exception de quelques gouvernorats ayant des activités touristiques intenses). Ceci peut expliquer en partie les graves problèmes sociaux qui se sont récemment posés dans la région.

\section{d) Dépenses par tête et besoins fondamentaux}

Les dépenses par tête, prises comme indice de niveau de vie, ne rendent pas parfaitement compte de la couverture de besoins fondamentaux des personnes en Égypte, certains services de base étant gratuits. Il est possible d'évaluer ces services au moyen de prix fantômes appropriés, mais ceux-ci sont difficiles à évaluer de manière empirique. Il importe donc d'examiner séparément la satisfaction des besoins fondamentaux, et d'essayer de les analyser en corrélation avec le niveau de dépenses par tête.

51 L'accès à un enseignement minimal est un des critères importants d'évaluation de la satisfaction des besoins fondamentaux. Malheureusement, les résultats préliminaires de l'enquête sur les revenus et dépenses de 1990/91 ne fournissent pas d'information sur le niveau d'éducation des membres du ménage. Les informations les plus récentes dont nous disposons sont les taux d'analphabétisme par gouvernorat, tirés du recensement de 1986. Le taux d'analphabétisme varie considérablement d'un gouvernorat à l'autre. Dans les régions urbaines des gouvernorats de Haute-Égypte, exception faite de Giza et d'Assouan, les taux d'analphabétisme dépassent la moyenne nationale. Il semble que les indices de pauvreté correspondent exactement aux taux d'analphabétisme. Le taux au Fayoum est le plus élevé, suivi par Sohag (tableau A11 de l'annexe - non reproduit; ndlr). Parmi les gouvernorats de la Basse-Égypte, Kafr alCheikh et Menoufiya ont les taux d'analphabétisme les plus élevés, et Le Caire le moins élevé, suivi par Isma'iliyya et Port-Saïd. 
52 L'enseignement a davantage porté ses fruits dans les régions urbaines que dans les régions rurales, où les taux d'analphabétisme s'élèvent à $35,16 \%$ et $61,29 \%$ respectivement. Que l'on considère les populations rurales ou urbaines, les taux d'analphabétisme de la plupart des gouvernorats de Haute-Égypte sont au-dessus de la moyenne nationale, exception faite de Giza et d'Assouan. En Basse-Égypte, Kafr alCheikh et Menoufiya sont les seuls gouvernorats dont les taux d'analphabétisme dépassent la moyenne nationale ; ils sont les plus faibles à Damiette.

53 Autre dimension de la pauvreté : l'insuffisance des services sanitaires. À. Badran ${ }^{9}$ a examiné, en 1987/88, les dépenses publiques de santé des différents gouvernorats : les plus fortes dépenses par tête dans ce domaine sont celles des gouvernorats «frontaliers» du Sud Sinaï, de la Nouvelle Vallée, de la Mer Rouge, de Matrouh et d'Assouan. Cela s'explique peut-être par leur densité démographique relativement faible. Damiette et la zone du Canal suivent. Les dépenses publiques par tête les plus faibles en matière de santé sont celles de Minya, Sohag, Beheira et Dakahlia. Le Caire et Alexandrie ne sont pas favorisés dans ce domaine.

54 Si l'on passe en revue les taux d'analphabétisme et les dépenses publiques de santé, il apparaît que la répartition régionale de la pauvreté, mesurée aux dépenses par tête des membres des ménages, correspond largement à celle de la satisfaction des besoins fondamentaux en matière d'enseignement et de santé.

\section{Évolution de la pauvreté dans le temps (1974/75 -1990/91)}

55 Àl'aide des résultats de l'enquête sur les budgets familiaux de 1974/75 et 1981/82 et des résultats préliminaires de l'enquête sur les revenus et dépenses effectuée en 1990/91, l'on a tenté d'évaluer l'évolution de la pauvreté en Égypte. Le niveau et la structure de la répartition des dépenses par tête ont été pris en considération. Les dépenses par tête au cours des trois périodes ont été évaluées en prix constants de 1981/82, par application de l'indice des prix à la consommation utilisé comme délateur. La moyenne des dépenses par tête en 1990/91 a été de 1.083,84 LE/an dans les régions urbaines et de 728,80 LE dans les régions rurales. En se basant sur les prix de 1981/82, ces chiffres passent à 269,51 LE et 186,36 LE respectivement, tandis que la moyenne des dépenses par tête en 1981/82 était de 277,17 LE et 188,00 LE respectivement, ce qui laisse apparaître une légère détérioration du niveau de vie entre 1981/82 et 1990/91.

56 Le niveau de vie des ménages s'est sensiblement amélioré entre 1974/75 et 1981/82. S'il s'est détérioré entre cette dernière date et 1990/91, il est cependant nettement supérieur actuellement à celui de 1974/75. Le tableau A13 en annexe indique la proportion de ménages par déciles, calculés en fonction du niveau de dépenses par tête, en se basant sur les prix de 1981/82. Il semble que les dépenses annuelles par tête des $10 \%$ des ménages urbains les plus pauvres étaient inférieures à 98,39 LE en 1974/75, puis se sont élevées à 133,16 LE en 1981/82 pour chuter à 118,83 LE en 1990/91. Ce schéma a prévalu dans tous les déciles dans les ménages urbains et ruraux.

57 Par ailleurs, la structure de la répartition des ménages selon leurs dépenses par tête montre une tendance au resserrement des disparités de revenus entre 1974/75 et 1981/82, puis une légère aggravation de ces disparités entre 1981/1982 et 1990/91, l'inégalité étant aujourd'hui inférieure à ce qu'elle était en 1974/75. Le tableau 6 indique que les dépenses des $20 \%$ des ménages urbains les plus pauvres représentaient 9,81\% de toutes les dépenses des ménages urbains en 1974/75; leur part dans les 
dépenses totales s'est élevée à $11,85 \%$ en $81 / 82$ pour retomber légèrement en dessous en $90 / 91$ (11,35\%), cependant que les dépenses des $20 \%$ des ménages les plus riches sont passées successivement de $37,41 \%$ à $33,32 \%$ et à $34,12 \%$ des dépenses totales de la population, leur part s'amenuisant sensiblement puis se rélargissant légèrement.

La structure de la répartition des ménages selon leurs dépenses par tête dans les régions rurales montre également une amélioration de 1974/75 à 1981/82, et une détérioration de 1981/82 à 1990/91, comme l'indique la diminution de la part des $20 \%$ des ménages les plus pauvres dans les dépenses totales de la population rurale, et l'élévation de la part des dépenses des $20 \%$ des ménages les plus riches.

Tableau 6 : part des $20 \%$ des ménages les plus pauvres et les plus riches dans les dépenses totales de la population

\begin{tabular}{|c|c|c|c|c|}
\hline $\begin{array}{c}\text { Année } \\
\text { d'enquête }\end{array}$ & \multicolumn{2}{|c|}{ Urbains } & \multicolumn{2}{c|}{ Ruraux } \\
\hline & $\begin{array}{c}\text { Les 20\% } \\
\text { les + pauvres }\end{array}$ & $\begin{array}{c}\text { Les 20\% } \\
\text { les plus riches }\end{array}$ & $\begin{array}{c}\text { Les 20\% } \\
\text { les + pauvres }\end{array}$ & $\begin{array}{c}\text { Les } 20 \% \\
\text { les plus riches }\end{array}$ \\
\cline { 2 - 5 } & & & & \\
$1974 / 75$ & 9,81 & 37,41 & 11,37 & 32,85 \\
$1981 / 82$ & 11,85 & 33,32 & 14,28 & 26,02 \\
$1990 / 91$ & 11,35 & 34,12 & 13,24 & 29,25 \\
\hline
\end{tabular}

Source : Calculs effectués sur la base des enquêtes sur les budgets des familles de 1974/75 et 1981/82, et l'enquête précédemment citée du CAPMAS.

Une autre manière d'évaluer la disparité des dépenses est de calculer des coefficients de Gini. Le tableau 7 donne ces coefficients pour la répartition des dépenses par tête des ménages urbains et ruraux. L'inégalité ainsi mesurée au niveau national s'est abaissée de 0,5397 en $1974 / 75$ à 0,4353 en $1981 / 82$, pour remonter à 0,4416 en $1990 / 91$, confirmant ainsi une amélioration suivie d'une légère détérioration au cours de la période examinée. Ce schéma a prévalu dans les régions urbaines et rurales. La disparité des dépenses semble être plus marquée dans les ménages urbains que dans les ménages ruraux.

Tableau 7 : coefficients de Gini par région et au niveau national

\begin{tabular}{|c|c|c|c|}
\hline Année deenquéte & R. urbaines & R. rurales & N. national \\
\hline $1974 / 75$ & 0,5187 & 0,5397 & 0,5397 \\
$1981 / 82$ & 0,4457 & 0,4139 & 0,4353 \\
$1990 / 91$ & 0,4432 & 0,4339 & 0,4416 \\
\hline
\end{tabular}

Source : même que tableau 6.

60 L'évolution de l'incidence, de l'intensité et des inégalités de pauvreté entre les trois enquêtes a été évaluée à l'aide des indices $\mathrm{P}_{0}, \mathrm{P}_{1}$ et $\mathrm{P}_{2}$ dans les régions rurales et 
urbaines. Les résultats figurent au tableau 8. L'incidence de la pauvreté baisse entre 1974/75 et 1981/82 dans les régions urbaines et s'élève ensuite légèrement en 1990/91. Le pourcentage de pauvres baisse, dans les régions urbaines, passant de $36,22 \%$ en 1974/75 à $26,18 \%$ en 1981/82, et se relève ensuite légèrement, atteignant $29,15 \%$. Les indices $\mathrm{P}_{1}$ et $\mathrm{P}_{2}$ suivent la même évolution, ce qui indique que la pauvreté était en baisse, tous critères confondus, entre 1974/75 et 1981/82, mais tend à s'élever en 1990/91.

Tableau 8 : Indices de pauvreté par région à diverses époques de l'enquête

\begin{tabular}{|c|c|c|c|c|c|c|}
\hline $\begin{array}{c}\text { Année } \\
\text { d'enquête }\end{array}$ & \multicolumn{3}{|c|}{ R. urbaines } & \multicolumn{3}{c|}{ R. rurales } \\
\hline & $P_{0}$ & $P_{1}$ & $P_{2}$ & $P_{0}$ & $P_{1}$ & $P_{2}$ \\
\cline { 2 - 7 } & & & & & & \\
$1974 / 75$ & 36,219 & 9,513 & 3,666 & 25,152 & 5,756 & 2,107 \\
$1981 / 82$ & 26,182 & 6,743 & 2,639 & 18,661 & 3,455 & 0,857 \\
$1990 / 91$ & 29,155 & 7,361 & 2,683 & 20,785 & 3,908 & 1,235 \\
\hline
\end{tabular}

Source : même que tableau 6.

61 Le graphique (non reproduit, ndlr) des distributions cumulées (pourcentages cumulés de la population située au-dessous des différents niveaux de consommation) à chaque enquête montre, aussi bien en région urbaine que rurale, que la courbe en 1981/82 se situe partout au-dessus de celles de 1990/91 et de 1974/75. La courbe en 1990/91 est très proche mais cependant inférieure à celle de 1981/82, tandis que celle de 1974/75 est la moins élevée. La pauvreté était à son apogée en 1974/75 et décline ensuite jusqu'à 1981/82, année du niveau de pauvreté le plus bas (tous critères confondus).

Par ailleurs, les tests statistiques indiquent que tous les indices de pauvreté étaient significativement plus élevés en 1974/75 qu'en 1981/82 et 1990/91, à un degré de confiance de $99 \%$. Les tests confirment une incidence accrue de la pauvreté $\left(\mathrm{P}_{0}\right)$ entre 1981/82 et 1990/91 dans les régions urbaines comme rurales, mais ne permettent pas d'inférer une différence significative de $\mathrm{P}_{1}$ et $\mathrm{P}_{2}$ entre ces deux dates.

\section{Les moyens financiers nécessaires pour combler l'écart de pauvreté}

63 Appliquant les indices $\mathrm{P}_{1}$ relatifs à l'écart du seuil de pauvreté, on a procédé à une évaluation préliminaire des moyens financiers nécessaires pour élever la moyenne des dépenses par tête des pauvres jusqu'au niveau du seuil de pauvreté, estimé à $2 / 3$ des dépenses moyennes par tête dans les différentes régions urbaines et rurales. En multipliant ces chiffres par le nombre total $d$ 'habitants concernés par région ( $d$ 'après les estimations de la population du CAPMAS pour 1990/91), on obtient le montant total des fonds nécessaires pour éliminer la pauvreté (tableau 9) : il s'élève à environ 1,9 milliard de LE soit 1,3 milliard pour les régions urbaines et 0,6 milliard pour les régions rurales. Il faudrait ainsi dégager, pour la Haute-Égypte, 756,2 millions de LE, pour la Basse-Égypte 590,3 millions, et pour Le Caire 238,9 millions. Pour ce qui est de l'extrême pauvreté, les fonds nécessaires à son élimination (ce qui amènerait la population concernée au seuil de pauvreté extrême fixé à $1 / 3$ des dépenses moyennes par tête) ne 
dépassent pas 213,8 millions LE (111,4 millions de LE pour les régions urbaines et 102,4 millions de LE pour les régions rurales).

Tableau 9 : Moyens financiers pour combler l'écart de pauvreté (par tête et globalement)

\begin{tabular}{|c|c|c|c|c|c|c|c|c|}
\hline \multirow[t]{3}{*}{ Régions } & \multicolumn{4}{|c|}{ Estimations maximales } & \multicolumn{4}{|c|}{ Estimations minimales } \\
\hline & \multicolumn{2}{|c|}{ par tête (LE) } & \multicolumn{2}{|c|}{ tolal (000 LE) } & \multicolumn{2}{|c|}{ par lète(LE) } & \multicolumn{2}{|c|}{ tolal (000 LE) } \\
\hline & ub.(1) & pur.(2) & urb.(3) & rur.(4) & uib.(5) & rur.(6) & urb.(7) & rur.(8) \\
\hline Le C & 34.30 & - & 238.934 & - & 4.58 & س & 31.904 & - \\
\hline Giza & 49.44 & 13.38 & 121.622 & 32.084 & 2.53 & 6.41 & 6.224 & 11.807 \\
\hline Alexandrie & 56.19 & - & 188.855 & - & 4.37 & 一 & 14.688 & 一 \\
\hline Cl Suez & 44.44 & - & 50.884 & - & 3.33 & - & 3.813 & - \\
\hline Bse-Egyple & 54.60 & 8.59 & 343.106 & 147.233 & 3.62 & 2.18 & 22.748 & 37.365 \\
\hline $\begin{array}{l}\text { Hie-Egyple } \\
\text { Ttes régions }\end{array}$ & 87.51 & 36.75 & 328.250 & 427.954 & 8.55 & 4.57 & $32.07 t$ & 52.218 \\
\hline $\begin{array}{l}\text { urbaines } \\
\text { Ttes régions } \\
\text { rurales }\end{array}$ & 53.19 & 18.99 & 1.271651 & 607.271 & 4.64 & 3.20 & 111.448 & $\begin{array}{c}- \\
10239\end{array}$ \\
\hline
\end{tabular}

En tout état de cause, le montant total des fonds requis pour éliminer la pauvreté et l'extrême pauvreté est inférieur aux fonds visés au titre des subventions et transferts directs inscrits au budget de 1990/91, qui se montaient à 6,2 milliards de $\mathrm{LE}^{10}$. Il importe donc de prévoir des subventions à l'intention des pauvres par le biais de transferts monétaires directs ou d'une distribution directe des produits de base; ainsi serait assurée à moindre coût une amélioration immédiate du niveau de vie des groupes cibles.

65 Appliquant la même démarche aux indices $P_{1}$ calculés pour 1981/82, on constate que les fonds qui étaient alors nécessaires pour éliminer la pauvreté étaient de l'ordre de 241 millions de LE pour les régions urbaines et de 106,7 millions de LE pour les régions rurales, soit un montant total de 347,7 millions de LE largement inférieur aux sommes allouées au titre des subventions inscrites au budget de 1981/82 (2,2 milliards LE) ${ }^{11}$.

\section{Structure des dépenses et sources de revenus des groupes pauvres}

La seconde partie de cette étude faisait état du degré de pauvreté en Égypte, de sa répartition régionale et son évolution, ainsi que des montants approximatifs nécessaires pour réduire la pauvreté en Égypte par le biais de transferts directs.

Nous tenterons à présent d'identifier les sources de revenus des pauvres, d'une part, et leur consommation telle que la reflète la structure de leurs dépenses, d'autre part. Nous établirons pour ce faire une distinction entre pauvres et non pauvres et comparerons ces deux groupes à l'ensemble des ménages enquêtes. Comme précédemment, la pauvreté sera définie en fonction de deux seuils distincts, le seuil le plus élevé $(722,56$ LE et 485,87 LE en ce qui concerne les dépenses par tête des ménages urbains et ruraux, 
respectivement) et le seuil le moins élevé, ou seuil d'extrême pauvreté (361,28 LE et $242,93 \mathrm{LE})$.

\section{Structure des dépenses par groupe de pauvreté}

Les tableaux A15 et A16 de l'annexe (non reproduits; ndlr) montrent deux modes de consommation des ménages urbains par groupe de pauvreté (le tableau A15 présente des pourcentages en colonne et le tableau A16, des pourcentages en ligne). Les produits alimentaires constituent le poste principal de dépenses des ménages : toutes catégories confondues, ils représentent $47,58 \%$ des dépenses totales des ménages urbains. Il existe une différence significative entre la part de dépenses alimentaires dans les dépenses totales des ménages pauvres et dans celles des ménages non-pauvres. Les premiers dépensent pour leur nourriture 57,03 \% de leur budget, contre seulement 45,46\% pour les seconds. L'impact de l'inflation a donc été plus sévère en Égypte pour les ménages pauvres que pour les autres, le prix des denrées alimentaires ayant augmenté plus fortement que l'indice global des prix à la consommation dans les régions urbaines.

Selon Besley et Kanbur ${ }^{12}$, ce qui importe à différents égards, ce n'est pas le poids d'un poste donné dans un budget, mais la part que les pauvres représentent dans l'ensemble des consommations monétaires. En d'autres termes, les pourcentages en ligne du tableau A16 (non reproduit ; ndlr) sont les plus révélateurs. Bien que les ménages urbains comptent $29,48 \%$ de ménages pauvres, les dépenses de ces derniers ne comptent que pour $18,27 \%$ dans la totalité des dépenses de l'ensemble des ménages, tandis que les ménages non-pauvres, qui représentent $70,52 \%$ du nombre total des ménages, dépensent $81,73 \%$ du montant total. La part des dépenses alimentaires des ménages pauvres, dans la totalité des dépenses alimentaires, est de $21,90 \%$, contre $78,10 \%$ pour les non-pauvres.

70 L'habillement représente la plus grande part des dépenses consacrées aux frais nonalimentaires dans le budget des ménages urbains non-pauvres, qui réalisent 84,30\% des dépenses totales d'habillement, n'en laissant que 15,70\% aux ménages pauvres. Les dépenses de logement représentent la plus grande part des dépenses non-alimentaires dans le budget des ménages pauvres. Ils affectent 9,98 \% de leur budget au logement mais leur part dans les dépenses totales de logement n'est que de $21,79 \%$ (les pourcentages respectifs pour les ménages non-pauvres sont de 8,01\% et 78,21\%).

71 Les dépenses de santé et de transports des ménages pauvres représentent 3,66\% et $2,49 \%$ de leurs dépenses, celles des non-pauvres $4,61 \%$ et $6,94 \%$. Les dépenses des pauvres en matière de santé et de transports représentent respectivement $15,06 \%$ et $7,41 \%$ de la totalité des dépenses au titre de ces deux rubriques. Ces proportions assez faibles s'expliquent en partie par le fait qu'il s'agit ici de services publics hautement subventionnés ou simplement gratuits. Les dépenses d'enseignement représentent respectivement 2,69 \% et 2,66 \% des budgets des ménages pauvres et non-pauvres. La part respective de chaque groupe par rapport à la totalité des dépenses d'enseignement est de $18,44 \%$ et de $81,56 \%$.

72 Le même schéma caractérise le comportement des ménages extrêmement pauvres. La part des dépenses alimentaires dans leur budget est plus élevée que celle des pauvres : elle s'élève à $62,12 \%$ contre $47,38 \%$ pour les autres ménages urbains. Pourtant, leurs dépenses ne représentent que $2,51 \%$ de la totalité des dépenses alimentaires. Bien que les ménages pauvres des régions rurales forment $20,88 \%$ du nombre total des ménages

Égypte/Monde arabe, 12-13| 1993 
couverts par l'enquête de 90/91, leurs dépenses ne représentent que $15,22 \%$ des dépenses totales (tableaux A17 et A18 de l'annexe - non reproduits; ndlr). Chez les ménages ruraux pauvres, l'alimentation représente $64,49 \%$ du budget, et chez les ménages non-pauvres, 55,79\%. Ce pourcentage est aussi plus élevé que chez les ménages urbains pauvres et très pauvres, ce qui s'explique comme suit : d'une part, les dépenses par tête sont relativement moins élevées dans les régions rurales; d'autre part, les prix des denrées alimentaires sont globalement plus élevés à la campagne qu'en ville, dans la mesure où les denrées subventionnées sont largement plus disponibles dans les zones urbaines. En zone rurale, l'alimentation des ménages pauvres ne représente que $17,18 \%$ de ce poste pour l'ensemble des ménages. Les dépenses de logement représentent la plus grande part des frais non-alimentaires des ménages ruraux pauvres et non-pauvres, part qui ne représente elle-même que 15,35 \% du montant total alloué au logement. Quant à la part des dépenses en matière de santé et de transport dans les budgets des ménages ruraux pauvres, elle est sensiblement inférieure à celle des ménages urbains pauvres $(2,71 \%$ et $1,94 \%$ contre $3,66 \%$ et $2,49 \%)$.

73 De même qu'en secteur urbain, le schéma décrit ci-dessus s'applique également aux ménages ruraux très pauvres, qui consacrent à l'alimentation une plus large part de leur budget $(69,23 \%)$, part qui représente une fraction des dépenses totales alimentaires encore plus faible que ce n'était le cas pour les urbains très pauvres $(2,24 \%$ contre $4,94 \%)$.

74 De manière surprenante, les cigarettes constituent un poste de dépenses dont le poids relatif est très élevé dans le budget des ménages pauvres et non pauvres; toutefois, il est plus élevé dans le budget des premiers que dans celui des seconds, aussi bien dans les régions urbaines que dans les régions rurales $(5,13 \%$ et $4,50 \%$ pour les ménages pauvres urbains et ruraux respectivement, contre $3,71 \%$ et $4,26 \%$ pour les ménages non pauvres).

\section{Source de revenus des groupes pauvres}

Procédons à présent à l'analyse des sources de revenus dans l'économie égyptienne, par groupe de pauvreté. Les tableaux A19 et A20 en annexe présentent les sources de revenus par groupes de pauvreté en ayant recours aux seuils de pauvreté les plus élevés et les plus bas pour les ménages urbains. Les salaires sont de toute évidence la source la plus importante de revenus chez les ménages urbains $(53,01 \%$ de la totalité de leurs revenus dans l'échantillon). Les revenus d'un emploi indépendant non-agricole $(22,35 \%$, cf. tableau A20) sont la deuxième source; suivent d'autres revenus représentant $11,96 \%$ de l'ensemble des sources, notamment ceux de quelques activités formelles et informelles.

En ce qui concerne les sources de revenus des pauvres dans les régions urbaines, leur classement par ordre d'importance est analogue à celui qui prévaut au niveau national bien que leur importance relative diffère, comme l'indique le pourcentage de chaque source de revenus par rapport à la totalité des revenus, par groupe de ménages. Les salaires sont la source principale de revenus - $56,85 \%$ du revenu total des ménages pauvres et $52,08 \%$ de celui des ménages non pauvres. Bien que les premiers représentent $29,48 \%$ du nombre total de ménages de l'échantillon, leurs salaires ne dépassent pas $20,95 \%$ de la totalité des revenus. De même, les revenus d'un emploi 
indépendant non agricole forment $20,24 \%$ des revenus des pauvres, mais ne représentent que $17,69 \%$ des revenus de cette catégorie au niveau national. Les revenus agricoles représentent $7,11 \%$ des revenus des ménages pauvres, mais seulement $4,88 \%$ des revenus des non pauvres. Les ménages urbains pauvres perçoivent - essentiellement au titre du fermage - 26,20\% du revenu agricole total. Enfin, si nous passons en revue la part qui revient aux ménages urbains pauvres dans toutes les catégories de revenus, nous pouvons conclure que la cause la plus importante de la disparité de revenus entre pauvres et non pauvres réside dans les autres sources, suivies des revenus tirés d'un emploi indépendant non agricole. Les revenus fonciers et de fermage sont des causes moins importantes de disparité, ce qui souligne l'efficacité des lois visant à contrôler les loyers en vue de neutraliser les avantages de la propriété foncière ou terrienne. Cela se reflète dans la part relativement élevée des ménages pauvres dans le revenu foncier ou agricole.

Ainsi, un accroissement marginal de 100 LE des revenus agricoles sera réparti entre 26 LE revenant aux ménages pauvres et $74 \mathrm{LE}$ aux non pauvres, tandis qu'un accroissement marginal de 100 LE du revenu total des ménages sera réparti entre 19,5 LE pour les ménages pauvres et 80,5 LE pour les non pauvres. Un accroissement semblable des salaires et des revenus fonciers sera réparti approximativement entre 20 pour les pauvres et 80 pour les non pauvres. Les propriétaires terriens devraient incontestablement retenir l'attention dans tout programme tendant à réduire la pauvreté.

78 Le classement par ordre d'importance des sources de revenus des ménages très pauvres est semblable à celui des pauvres. Toutefois, la part des salaires dans les revenus des très pauvres tombe à $49,75 \%$ contre $56,85 \%$ pour les pauvres, et la perspective d'un revenu découlant d'autres ressources est inexistante. Par ailleurs, le pourcentage d'autres catégories de revenus (agricole, non-agricole, foncier et autres) est en hausse, ce qui souligne le fait que quelques propriétaires terriens et fonciers tombent dans la catégorie des ménages très pauvres de l'échantillon. Les revenus agricoles sont la première source de revenus des ménages ruraux $(48,94 \%$ pour l'ensemble des ménages, $44,42 \%$ pour les pauvres et $49,79 \%$ pour les non pauvres). Les salaires forment la seconde source: $34,85 \%$ des revenus des ruraux pauvres, $24,89 \%$ de ceux des non pauvres et $26,47 \%$ de l'ensemble (tableau A21 et A22). Les autres sources de revenus, classées par ordre dégressif, sont l'emploi indépendant non-agricole, la propriété foncière et enfin diverses sources (tableau A22).

Notons que les salaires semblent être équitablement répartis entre les divers ménages ruraux, si l'on en juge à la part des pauvres dans l'ensemble des ménages $(20,88 \%$ en $1990 / 91)$ et dans l'ensemble des salaires (20,87 \%). Cependant, leur part dans l'ensemble des revenus ruraux ne dépasse pas $15,84 \%$ à cause de la répartition inégale des autres sources de revenus.

La répartition du revenu total en fonction des sources, entre pauvres et non pauvres, peut éclairer sur les moyens de réduire plus efficacement la pauvreté. Ainsi que nous l'avons souligné précédemment, si le montant total des salaires dans les régions rurales était majoré de $100 \mathrm{LE}$, le revenu du pauvre augmenterait marginalement de 20,86 LE, tandis que si l'on augmentait la totalité des revenus provenant d'autres sources d'un même montant, le revenu du pauvre s'élèverait de 6,09 LE à 14,38 LE selon la catégorie de revenus augmentée. 
81 Pour les très pauvres, l'importance relative des diverses sources de revenus diffère sensiblement de ce que nous venons de décrire. Contrairement aux autres ménages ruraux, leur source principale de revenus sont les salaires (44,91\%). Viennent ensuite les revenus agricoles $(23,93 \%)$ et autres, y compris les revenus d'activités informelles $(15,12 \%)$ (tableau A22). L'extrême pauvreté semble être le lot des paysans sans terre, de la main-d'oeuvre rurale occasionnelle et des salariés en général.

\section{Caractéristiques démographiques des couches pauvres}

Le niveau de l'enseignement, l'occupation, l'activité du chef de famille, la dimension du ménage sont les variables les mieux corrélées avec la pauvreté.

\section{Dimension des ménages}

La famille nombreuse caractérise les pauvres. L'enquête sur les revenus et dépenses de 1990/91 révèle des différences significatives dans la dimension moyenne des ménages pauvres et non pauvres. Le tableau 10 indique que la dimension moyenne des ménages pauvres est de 6,49 dans les régions urbaines et de 7,80 dans les régions rurales, tandis que les chiffres correspondants sont de 4,73 et de 6,31 pour les ménages non-pauvres. Au niveau national, la dimension moyenne est 5,25 pour les ménages urbains et 6,65 pour les ménages ruraux. Ces différences entre les dimensions des ménages sont encore plus marquées lorsqu'il s'agit d'extrême pauvreté. Les dimensions moyennes des ménages très pauvres sont de 7,39 et de 8,35 contre 5,18 et 6,63 pour les "autres " groupes urbains et ruraux respectifs.

Tableau 10 : Taille des ménages par groupes de pauvreté et par région (1990/91)

\begin{tabular}{|c|c|c|c|c|c|}
\hline Région & Pauvres & Non pauves & $\begin{array}{c}\text { Toutes } \\
\text { catégories }\end{array}$ & $\begin{array}{c}\text { Extrèmement } \\
\text { pauvres }\end{array}$ & Autres \\
\hline urbaines & 6,490 & 4,725 & 5,245 & 7,393 & 5,175 \\
rurales & 7,796 & 6,312 & 6,646 & 8,346 & 6,631 \\
\hline
\end{tabular}

Source : même que tableau 6

L'enquête de 1990/91 ne fournit malheureusement pas d'informations sur la répartition des ménages selon l'occupation, le niveau d'instruction ou l'activité du chef de ménage. Nous avons donc dû recourir aux résultats de l'enquête sur les budgets familiaux de 1991/92. Les seules données disponibles sont les répartitions des ménages selon ces variables et les dépenses du ménage, quelle que soit sa dimension. Les seuils de pauvreté sont ainsi fixés aux $2 / 3$ de la totalité des dépenses moyennes par ménage, à savoir 960,8 LE par an pour les ménages urbains et 726,3 LE pour les ménages ruraux ${ }^{13}$. Les indices de pauvreté calculés sur la base de ces seuils de pauvreté diffèreront probablement de ceux précédemment calculés. 


\section{Occupation des couches pauvres}

\section{famille. Les ménages dont le chef de famille est «inactif» ont les indices de pauvreté} les plus élevés. Dans cette catégorie, l'incidence de pauvreté $\mathrm{P}_{0}$ atteint $46,45 \%$ tandis que l'intensité de la pauvreté, mesurée en fonction de $\mathrm{P}_{1}$ est de l'ordre de $17,79 \%$ et l'indice $\mathrm{P}_{2}$ se situe à $9,87 \%$. La contribution de cette catégorie à l'ensemble de la pauvreté des ménages s'élève à $28,88 \%, 39,10 \%$ et $45,90 \%$ si l'on considère respectivement $\mathrm{P}_{0}, \mathrm{P}_{1}$ et $\mathrm{P}_{2}$, tandis que les ménages dont le chef de famille est classé comme "inactif» ne représentent que $21,08 \%$ des ménages. Nous pouvons donc conclure que selon tous les critères, les ménages dont le chef de famille est «inactif » souffrent de la pauvreté la plus aiguë. Voilà qui ne saurait surprendre, les personnes âgées et les handicapés relevant de cette même catégorie. Par l'incidence de pauvreté mesurée par $\mathrm{P}_{0}$, les ouvriers agricoles viennent au deuxième rang et le personnel du secteur des services au troisième; ce classement s'inverse en considérant $\mathrm{P}_{1}$ et $\mathrm{P}_{2}$. En outre, il semble que la disparité entre les pauvres, mesurée par $\mathrm{P}_{2}$, soit plus élevée parmi les travailleurs indépendants qu'elle ne l'est parmi les ouvriers agricoles. La contribution du personnel de service à l'ensemble des indices de pauvreté est plus élevée que celle de ménages agricoles. Cette contribution dépasse la part relative respective des ménages engagés dans ces occupations par rapport à la totalité des ménages qui ont fait l'objet de l'analyse. Enfin, malgré des indices de pauvreté relativement bas, les ouvriers des transports et de la production apportent à la pauvreté globale une contribution relativement élevée $\left(24,20 \%\right.$ en fonction de $\mathrm{P}_{0}, 17,24$ selon $\mathrm{P}_{1}$ et 14,73 selon $\mathrm{P}_{2}$ ), qui tient au nombre relativement important des ménages travaillant dans ces secteurs.

En tout état de cause, un programme efficace tendant à réduire la pauvreté dans les régions urbaines devrait mettre l'accent tout particulièrement sur les ménages dont le chef de famille est « inactif » et sur les ouvriers de la production et des transports. Dans les régions rurales (cf. tableau A24 en annexe), les indices de pauvreté les plus élevés sont ceux des ménages dont le chef de famille est « inactif ». Les indices les plus faibles sont ceux des ménages dont le chef de famille a un emploi «indépendant", y compris les travailleurs non qualifiés et occasionnels dans les régions rurales. Ces deux catégories de ménages représentent un pourcentage de la pauvreté nationale bien supérieur à leurs poids dans la population, ce qui indique clairement leur niveau élevé de pauvreté. Les ménages dont le chef de famille est ouvrier agricole viennent au troisième rang de l'incidence de la pauvreté ; toutefois, il s'agit de la catégorie la plus nombreuse dans l'échantillon $(51,76 \%)$ et, partant, elle appelle une plus grande attention.

En conclusion, dans l'élaboration d'un programme tendant à réduire la pauvreté, l'accent doit être mis sur les ménages dont le chef de famille est soit «inactif», soit travailleur indépendant soit ouvrier agricole. 


\section{Activité des pauvres} (« autres») sont pauvres, selon l'indice $\mathrm{P}_{0}$. Les indices $\mathrm{P}_{1}$ et $\mathrm{P}_{2}$ sont également les plus élevés pour cette catégorie de ménages (respectivement 30,64 et 19,04). Toutefois, la contribution de ces ménages à la pauvreté globale n'est pas la plus élevée, étant donné leur part relativement faible, à savoir 4,16\% des ménages ruraux qui ont fait l'objet de notre analyse. $\mathrm{PO}, \mathrm{P}_{1}$ et $\mathrm{P}_{2}$ s'élèvent respectivement à $12,54 \%, 22,51 \%$ et $24,46 \%$. Les valeurs élevées de a signifient qu'on a accordé davantage de poids aux ménages les plus pauvres de chaque catégorie, ce qui suppose qu'un assez grand nombre de ménages de cette catégorie sont parmi les plus pauvres. L'agriculture est l'activité que les indices de pauvreté placent en second, mais c'est elle qui contribue le plus largement à la pauvreté globale, à la hauteur de $66,64 \%$ pour $\mathrm{PO}, 59,33 \%$ pour $\mathrm{P} 1$ et $55,84 \%$ pour $\mathrm{P} 2$, à cause de sa prépondérance parmi les ménages ruraux. Une attention particulière doit être accordée aux ménages engagés dans des activités agricoles et non classées, car ils contribuent pour plus de $50 \%$ à la pauvreté rurale globale.

\section{Niveau de l'enseignement des pauvres}

En corrélation étroite avec l'occupation, l'enseignement est une variable importante qui influe sur la pauvreté. Il est également un bon indicateur de la capacité des ménages pauvres à satisfaire leurs besoins fondamentaux.

Les tableaux A27 et A28 fournissent les indices de pauvreté selon le niveau d'instruction du chef de famille et la région, urbaine ou rurale. De toute évidence, les ménages dont le chef de famille est analphabète ont les indices de pauvreté les plus élevés. Dans cette catégorie de ménages, l'incidence de la pauvreté est de $41,38 \%$ en ville et de 51,15 \% à la campagne. L'intensité de la pauvreté mesurée par $\mathrm{P}_{1}$ est de 15,9\% et $25,23 \%$ respectivement pour les ménages urbains et ruraux. Donnant plus de poids aux ménages les plus pauvres, l'indice $\mathrm{P}_{2}$ des ménages dont le chef de famille est 
analphabète atteint $9,12 \%$, et $16,98 \%$ respectivement pour les régions urbaines et rurales. La fraction des ménages pauvres qui vient au deuxième rang est celle dont le chef de famille n'a reçu qu'une instruction primaire. Entrent dans cette catégorie $70,35 \%$ et $93,42 \%$ des ménages urbains et ruraux respectivement, mais leur contribution à la pauvreté nationale dépasse ces pourcentages pour atteindre $84,18 \%$ et $96,25 \%$ mesurés par $\mathrm{P}_{0}, 88,59 \%$ et $95,76 \%$ mesurés par $\mathrm{P}_{1}$ et enfin, $89,40 \%$ et $95,4 \%$ mesurés par $\mathrm{P}_{2}$, pour les ménages urbains et ruraux respectivement. Ces chiffres soulignent la gravité de la pauvreté de cette catégorie et la nécessité de la considérer comme prioritaire.

\section{Résumé}

L'objet de cette étude était d'esquisser le profil de la pauvreté en Égypte en fournissant des informations sur la localisation, la structure de la consommation, les sources de revenus, les activités économiques et d'autres caractéristiques pertinentes de la population étudiée. Sur le plan méthodologique, nous avons appliqué les concepts les plus récents pour définir et mesurer la pauvreté. En appliquant ces concepts, nous avons tenu compte du caractère incertain des données. Les résultats auxquels nous sommes parvenus sont présentés comme une première étude des statistiques disponibles. Nous nous sommes essentiellement fondés sur les informations préliminaires fournies par l'enquête sur les revenus et dépenses de 1990/91 et les résultats de l'enquête sur les budgets familiaux de 1981/82.

Deux seuils de pauvreté ont été fixés, l'un pour les régions urbaines et l'autre pour les régions rurales. L'un des seuils a été fixé à $2 / 3$ des dépenses moyennes par tête pour chaque région, et le second à 1/3. L'application du seuil de pauvreté le plus élevé a donné le profil de pauvreté suivant:

1. le niveau de vie (mesuré en fonction des dépenses par tête) est plus élevé dans les régions urbaines que dans les régions rurales. Toutefois, l'incidence, l'intensité et la gravité de la pauvreté sont relativement moindres dans les secondes que dans les premières, quel que soit l'indice de pauvreté. Plus de $29 \%$ de ménages urbains sont relativement pauvres, contre $21 \%$ de ménages ruraux ;

2. l'incidence de pauvreté varie considérablement selon la région et le gouvernorat et au sein de chaque région. Elle est maximale en Haute-Égypte (Fayoum, Sohag et Assiout). Cela reflète des différences en matière de productivité agricole et de concentration industrielle, situation aggravée par de faibles niveaux d'instruction et des service de santé insuffisants. Le développement du tourisme constitue un net facteur d'amélioration des niveaux de vie à Giza et à Assouan. Enfin, parmi les gouvernorats de Basse-Égypte, Isma'iliyya et Damiette sont les plus avantagés ;

3. selon les indicateurs que constituent le niveau d'instruction et la santé, les régions urbaines sont plus avantagées que les régions rurales, et la Basse-Égypte l'est davantage que la HauteÉgypte ;

4. les pauvres consacrent une plus grande part de leur budget (de $57 \%$ à 64\%) à la nourriture. Cela reflète un plus fort impact de l'ajustement structurel sur cette catégorie, les prix des produits alimentaires ayant augmenté, en 1990/91 et 1991/92, à un rythme dépassant l'indice des prix à la consommation ;

5. la pauvreté est associée aux salaires dans les régions urbaines et à l'agriculture dans les régions rurales ; 
6. les indices de pauvreté sont plus élevés dans les ménages dont le chef de famille est classé «inactif» (les veuves, les personnes âgées, les malades et les handicapés sont particulièrement dépendants des transferts directs de revenus). Dans les régions urbaines, la pauvreté est également associée à l'emploi dans l'agriculture, les services, le secteur gouvernemental, les activités peu qualifiées, marginales ou indépendantes. Dans les régions rurales, les pauvres sont pour la plupart des fermiers non propriétaires, de petits propriétaires terriens et des travailleurs agricoles;

7. le niveau d'instruction est inversement proportionnel à la pauvreté. Les ménages qui contribuent le plus au maintien du niveau national de pauvreté sont ceux dont le chef de famille est analphabète ou n'a reçu qu'un enseignement minimal.

Le classement par ordre d'importance des indices de pauvreté, en ce qui concerne les régions et les autres caractéristiques économiques et démographiques des ménages, paraît solide quant au choix des indices et les seuils de pauvreté; cependant, l'incidence de l'extrême pauvreté est un peu plus élevée dans les régions rurales que dans les régions urbaines. Bien que l'incidence de la pauvreté se situe au-dessous de la moyenne au Caire et à Alexandrie, une extrême pauvreté existe néanmoins dans ces deux villes, probablement en raison de la migration des régions rurales. Cette observation appelle une étude approfondie.

En outre, le niveau de vie s'est sensiblement amélioré entre 1974/75 et 1981/82 mais s'est légèrement détérioré en 1990/91. La structure de la répartition des dépenses s'est également améliorée mais s'est ensuite légèrement détériorée au cours de la même période.

99 Les fonds nécessaire pour éliminer la pauvreté et l'extrême pauvreté sont de loin inférieurs au fardeau actuel des subventions imputées directement sur le budget de l'État, ce qui incite à prendre des mesures pour réduire la pauvreté.

Pour finir, le profil préliminaire de la pauvreté esquissé dans cette étude permet d'évaluer dans quelle mesure les structures sectorielles et régionales des changements économiques peuvent avoir un impact sur la pauvreté globale en Égypte. Ce profil indique également que toute politique de réforme améliorant relativement le niveau de vie des salariés, des travailleurs agricoles et des ménages dont le chef de famille est classé «inactif est en mesure de réduire la pauvreté collective dans le cadre de l'ensemble de l'économie. 
Tableau A7 : Indices de pauvreté par gouvemorats urbains en 1990/91 (seuil de pauvreté $=722,56$ LE)

\begin{tabular}{|c|c|c|c|c|c|c|}
\hline \multirow{2}{*}{ Gouvemorat } & \multicolumn{3}{|c|}{ Indices de paurreté } & \multicolumn{3}{|c|}{ Contribution * } \\
\hline & PO & P1 & P2 & PO & P1 & $\mathbf{P 2}$ \\
\hline Le Caire & 19.525 & 4.747 & 1.656 & 19.125 & 18.035 & 17.260 \\
\hline Alexandrio & 27.963 & 7.776 & 3.169 & 12.297 & 13.544 & 15.096 \\
\hline Port-Said & 17.647 & 3.507 & 1.089 & 0.972 & 0.769 & 0.655 \\
\hline Suez & 44.444 & 12.182 & 4.227 & 1.945 & 2122 & 2.020 \\
\hline Damiette & 13.954 & 2.821 & 0.819 & 0.486 & 0.405 & 0.312 \\
\hline Dakahliya & 17.073 & 4.215 & 1,811 & 2.836 & 2.787 & 3.286 \\
\hline Charqiyja & 36.691 & 6.649 & 1.849 & 4.133 & 2.981 & 2.274 \\
\hline Oaliubiyya & 39.768 & 8.903 & 2.942 & 8.347 & 7.467 & 6.432 \\
\hline K. at-Chaykh & 32.584 & 10.263 & 3.907 & 2.350 & 2.946 & 3.077 \\
\hline Gharbiyya & 25.424 & 5.783 & 2.158 & 3.647 & 3.301 & 3.379 \\
\hline Menufyya & 48.544 & 13.514 & 5.326 & 4.052 & 4.490 & 4.855 \\
\hline Beheira & 37.333 & 8.367 & 2.743 & 4.538 & 4.048 & 3.641 \\
\hline smailiyya & 10.811 & 2.202 & 0.556 & 0.324 & 0.263 & 0.182 \\
\hline Giza & 23.867 & 6.842 & 2.397 & 9.076 & 9.556 & 9.184 \\
\hline Beni-Suef & 35.294 & 7.942 & 2.694 & 1.945 & 1.742 & 1.621 \\
\hline Fayoum & 66.667 & 21.414 & 9.181 & 4.052 & 5.180 & 6.093 \\
\hline Minya & 33.433 & 9.939 & 3.727 & 3.647 & 4.071 & 4.189 \\
\hline Assioul & 49.606 & 15.578 & 6.447 & 5.105 & 6.381 & 7.245 \\
\hline Sohag & 39.167 & 15.018 & 5.291 & 5.754 & 5.813 & 5.619 \\
\hline Cena & 38.938 & 7.891 & 2.417 & 3.566 & 2.876 & 2.417 \\
\hline Aswan & 27.586 & 4.342 & 1.005 & 1.297 & 0.812 & 0.516 \\
\hline Mairuh & 14.286 & 2.782 & 0.770 & 0.567 & 0.440 & 0.334 \\
\hline Ttes zones & 29.297 & 7.361 & 2.683 & 100 & 100 & 100 \\
\hline
\end{tabular}

Source : résultats préliminaires de l'enquête 1990/91 sur les revenus et dépenses, CAPMAS, 1992.

* Contribution d'un gouvernorat à l'indice national de pauvreté = (indice de pauvreté du gouvernorat)

(\% nombre de ménages)/(indice national de pauvreté). 
Tableau A8 : Indices de pauvreté par gouvemorats urbains en 1990/91 (seuil de pauvreté $=361,28$ LE)

\begin{tabular}{|c|c|c|c|c|c|c|}
\hline \multirow{2}{*}{ Gouvemorat } & \multicolumn{3}{|c|}{ Indices de pauvreté } & \multicolumn{3}{|c|}{ Contribution } \\
\hline & PO & P1 & P2 & PO & P1 & P2 \\
\hline Le Caire & 4.075 & 1.267 & 0.540 & 24.490 & 27.699 & 28.685 \\
\hline Alexandrie & 5.556 & 1.209 & 0.507 & 15.306 & 12.112 & 12.360 \\
\hline Port-Saidd & 2.941 & 1.296 & 0.996 & 1.020 & 2.142 & 3.055 \\
\hline Suez & 3.794 & 0.574 & 0.089 & 1.020 & 0.575 & 0.216 \\
\hline Damiette & 2.326 & 1.096 & 0.517 & 0.510 & 0.875 & 1.002 \\
\hline Dakahlyya & 2.439 & 0.684 & 0.160 & 2.551 & 2.601 & 1.479 \\
\hline Charqiyya & 1.439 & 0.420 & 0.139 & 1.020 & 1.084 & 0.869 \\
\hline Qaliubiya & 3.681 & 0.595 & 0.130 & 5.102 & 2.856 & 0.522 \\
\hline K. al-Chaykh & 3.371 & 0.666 & 0.182 & 1.531 & 1.200 & 0.730 \\
\hline Gharbiya & 5.370 & 2.031 & 0.973 & 5.102 & 6.669 & 7.757 \\
\hline Merwinya & 4.854 & 0.750 & 0.192 & 2.551 & 1.434 & 0.892 \\
\hline Beheira & 4.667 & 1.806 & 0.983 & 3.571 & 3.026 & 6.653 \\
\hline Ismailiyya & 0.000 & 0.000 & 0.000 & 0.000 & 0.000 & 0.000 \\
\hline Giza & 3.233 & 0.700 & 0.237 & 7.143 & 5.625 & 4.632 \\
\hline Beni-Suef & 5.882 & 2.869 & 1.354 & 2.041 & 3.619 & 4.154 \\
\hline Fayoum & 13.333 & 5.887 & 2.985 & 5.102 & 8.192 & 10.099 \\
\hline Minya & 5.512 & 1.429 & 0.549 & 3.571 & 3.367 & 3.144 \\
\hline Assiout & 10.236 & 1.711 & 0.532 & 6.633 & 4.031 & 3.049 \\
\hline Sohag & 12.500 & 2.946 & 0.943 & 7.653 & 6.559 & 5.107 \\
\hline Dena & 6.195 & 1.936 & 0.865 & 3.571 & 4.059 & 4.408 \\
\hline Aswan & 1.724 & 0.352 & 0.072 & 0.510 & 0.379 & 0.188 \\
\hline Matruh & 0.000 & 0.000 & 0.000 & 0.000 & 0.000 & 0.000 \\
\hline Ttes zones & 4.653 & 1.280 & 0.526 & 100 & 100 & 100 \\
\hline
\end{tabular}

Source : résultats préliminaires de l'enquête 1990/91 sur les revenus et dépenses, CAPMAS, 1992.

* Contribution d'un gouvernorat à l'indice national de pauvreté = (indice de pauvreté du gouvernorat)

(\% nombre de ménages)/(indice national de pauvreté). 
Tableau A9 : Indices de pauvreté par gouvemorats ruraux en 1990/91 (seuil de pauvreté $=464.87$ LE)

\begin{tabular}{|c|c|c|c|c|c|c|}
\hline \multirow{2}{*}{ Gouvertiorats } & \multicolumn{3}{|c|}{ Indices de pauvreté } & \multicolumn{3}{|c|}{ Contribution * } \\
\hline & PO & P1 & $\mathrm{P} 2$ & PO & P1 & $\mathrm{P} 2$ \\
\hline Damielte & 1.695 & 0.310 & 0.057 & 0.163 & 0.158 & 0.091 \\
\hline Dakahlyya & 9.667 & 1.559 & 0.379 & 4.723 & 4.053 & 3.117 \\
\hline Charqiyya & 22.559 & 4.137 & 1.283 & 10.912 & 10.645 & 10.533 \\
\hline Qailubinya & 4.138 & 0.681 & 0.148 & 0.977 & 0.855 & 0.588 \\
\hline K. atChaykh & 5.970 & 0.488 & 0.071 & 1.300 & 0.989 & 0.260 \\
\hline Gharbiyya & 15.812 & 3.108 & 0.928 & 6.026 & 6.301 & 5.958 \\
\hline Menufirya & 7.317 & 1.092 & 0.257 & 2.443 & 1.939 & 1.442 \\
\hline Beheira & 18.039 & 3.044 & 0.788 & 7.492 & 6.724 & 5.510 \\
\hline isma'iliyya & 0.000 & 0.000 & 0.000 & 0.000 & 0.000 & 0.000 \\
\hline Giza & 16.667 & 2.753 & 0.706 & 3.909 & 3.435 & 2.787 \\
\hline Beni-Suel & 28.829 & 5.876 & 1.855 & 5.212 & 5.485 & 5.647 \\
\hline Fayoum & 45.378 & 13.194 & 4.908 & 8.795 & 13.602 & 16.018 \\
\hline Wina & 49.580 & 10.103 & 3.172 & 19.218 & 21.449 & 20.705 \\
\hline Assiout & 33.702 & 9.153 & 3.155 & 9.935 & 14.353 & 15.661 \\
\hline Sohay & 36.191 & 7.483 & 2.461 & .12 .378 & 13.614 & 14.172 \\
\hline Ora & 9.714 & 1.686 & 0.518 & 2.769 & 2.557 & 2.487 \\
\hline Aswan & 6.780 & 0.786 & 0.164 & 0.652 & 0.402 & 0.263 \\
\hline Matruh & 32.203 & 4.843 & 0.953 & 3.095 & 2.475 & 1.542 \\
\hline Ttes zones & 20.785 & 3.908 & 1.234 & 100 & 100 & 100 \\
\hline
\end{tabular}

Source : résultats préliminaires de l'enquête 1990/91 sur les revenus et dépenses, CAPMAS, 1992.

* Contribution d'un gouvernorat à l'indice national de pauvreté = (indice de pauvreté du gouvemorat)

(\% nombre de ménages)/(indice national de pauvreté). 
Tableau A10 : Indices de pauvreté par gouvernorats ruraux en 1990/91 (seuil de pauvreté $=484.87$ LE)

\begin{tabular}{|c|c|c|c|c|c|c|}
\hline \multirow{2}{*}{ Gowernorats } & \multicolumn{3}{|c|}{ Indices de pauvrelé } & \multicolumn{3}{|c|}{ Contribution * } \\
\hline & Po & P1 & P2 & PO & P1 & $P 2$ \\
\hline Damielte & 1.695 & 0.992 & 0.581 & 0.671 & 1.502 & 2.132 \\
\hline Dabahlyya & 0.667 & 0.124 & 0.073 & 1.342 & 0.957 & 1.361 \\
\hline Charqiya & 5.387 & 1.790 & 0.963 & 10.811 & 13.759 & 17.921 \\
\hline Oailubiyya & 1.379 & 0.212 & 0.060 & 1.342 & 0.789 & 0.542 \\
\hline K. al-Chaykh & 0.746 & 0.145 & 0.028 & 0.671 & 0.498 & 0.234 \\
\hline Gharbiyya & 5.128 & 1.851 & 0.799 & 8.054 & 11.115 & 11.630 \\
\hline Menufiya & 4.878 & 1.381 & 0.666 & 6.711 & 7.263 & 8.494 \\
\hline Beheira & 1.177 & 0.266 & 0.074 & 2.013 & 1.741 & 1.167 \\
\hline Ismatliya & 0.000 & 0.000 & 0.000 & 0.000 & 0.000 & 0.000 \\
\hline Giza & 7.639 & 2.638 & 1.101 & 7.383 & 9.747 & 9.868 \\
\hline Beni-Suet & 9.910 & 2.574 & 0.921 & 7.383 & 7.332 & 6.366 \\
\hline Fayoum & 13.445 & 4.233 & 1.978 & 10.738 & 12.926 & 14.651 \\
\hline Mrya & 7.143 & 1.167 & 0.323 & 11.409 & 7.129 & 4.788 \\
\hline Assiout & 8.840 & 1.731 & 0.455 & 10.738 & 8.037 & 5.129 \\
\hline Sohag & 8.095 & 1.568 & 0.547 & 11.409 & 8.451 & 7.144 \\
\hline Oena & 5.714 & 1.727 & 0.787 & 6.711 & 7.754 & 8.575 \\
\hline Aswan & 3.390 & 0.530 & 0.119 & 1.342 & 0.807 & 0.435 \\
\hline Matruh & 3.390 & 1.396 & 0.655 & 1.342 & 2.114 & 2.407 \\
\hline Ties zones & 5.044 & 1.319 & 0.544 & 100 & 100 & 100 \\
\hline
\end{tabular}

Source : résultats préliminaires de l'enquête 1990/91 sur les revenus et dépenses, CAPMAS, 1992.

* Contribution d'un gouvemorat à l'indice national de pauvreté = (indice de pauvreté du gouvemorat)

(\% nombre de ménages)/(indice national de pauvreté). 
Tableau A12 : Dépenses de santé totales et courantes par tête et par gouvernorat (1987/88)

\begin{tabular}{|c|c|c|}
\hline Gouvemorat & $\begin{array}{c}\text { Dépenses totales } \\
\text { par tốte }\end{array}$ & $\begin{array}{c}\text { Dépenses courantes } \\
\text { par têtie }\end{array}$ \\
\hline Le Caire & 7.20 & 1.62 \\
\hline Alexandrie & 9.82 & 1.31 \\
\hline Port-Sald & 19.06 & 3.66 \\
\hline Suez & 14.36 & 2.75 \\
\hline Damiette & 39.22 & 7.05 \\
\hline Dakehliyya & 6.95 & 0.91 \\
\hline Charqiyya & 7.65 & 0.88 \\
\hline Qaliubiyja & 7.68 & 1.65 \\
\hline K. al-Chaykh & 7.20 & 1.20 \\
\hline Gharbiyya & 9.07 & 1.52 \\
\hline Menufiyya & 8.29 & 1.18 \\
\hline Beheira & 6.97 & 0.90 \\
\hline Ismáliyya & 13.90 & 2.44 \\
\hline Giza & 8.7 & 1.99 \\
\hline Beni-Suel & 11.15 & 1.60 \\
\hline Fayoum & 8.07 & 1.24 \\
\hline Minya & 6.43 & 1.15 \\
\hline Assiout & 8.46 & 1.13 \\
\hline Sohag & 6.86 & 0.95 \\
\hline Cena & 7.71 & 1.38 \\
\hline Aswan & 15.50 & 3.13 \\
\hline Matruh & 21.88 & 4.22 . \\
\hline Nord Sinal & 23.64 & 4.22 \\
\hline Sud Sinat & 89.83 & 13.80 \\
\hline Mer Rouge & 33.94 & 6.14 \\
\hline Nouselie Vallé & 35.77 & 6.96 \\
\hline
\end{tabular}

Source : Badran A., « Structural adjustments in heaith sector to protect vulnerable groups ». The Third World Forum and UNICEF.

Tableau A13 : Dépenses proportionnelles des ménages par tête, en déciles (prix de 1981/92)

\begin{tabular}{|c|r|r|r|r|r|r|}
\hline \multirow{2}{*}{$\begin{array}{c}\text { nornbre } \\
\text { de mérages }\end{array}$} & \multicolumn{3}{|c|}{ Urbains } & \multicolumn{3}{c|}{ Ruraux } \\
\cline { 2 - 7 } & $74 / 75$ & $81 / 82$ & $90 / 91$ & $74 / 75$ & $81 / 82$ & $90 / 91$ \\
\hline $10 \%$ & 98,37 & 133,16 & 118,83 & 76,25 & 108,71 & 100,83 \\
$20 \%$ & 122,03 & 163,80 & 153,29 & 92,74 & 130,89 & 119,63 \\
$30 \%$ & 144,58 & 191,46 & 180,45 & 106,30 & 148,54 & 137,74 \\
$40 \%$ & 168,54 & 221,10 & 209,16 & 120,18 & 165,67 & 156,08 \\
$50 \%$ & 195,71 & 251,70 & 241,14 & 135,60 & 184,46 & 175,85 \\
$60 \%$ & 227,02 & 288,51 & 256,15 & 154,75 & 207,71 & 194,86 \\
$70 \%$ & 286,56 & 340,98 & 308,67 & 177,26 & 237,83 & 223,96 \\
$80 \%$ & 352,41 & 421,78 & 408,13 & 209,96 & 280,11 & 261,89 \\
$90 \%$ & 506,49 & 587,83 & 568,60 & 288,93 & 357,91 & 335,08 \\
& & & & & & \\
\hline
\end{tabular}

Source : enquête 1974/75 sur le budget des familles et l'enquête 1981/82 sur les revenus et dépenses, CAPMAS 
Tableau A19 : Origine des revenus des ménages urbains par groupes de pauvreté (1990/91)

\begin{tabular}{|l|r|r|r|r|r|r|r|r|}
\hline Groupes & Salaizes & \multicolumn{1}{c|}{$\begin{array}{c}1 \\
\text { (a) }\end{array}$} & \multicolumn{1}{c|}{$\begin{array}{c}1 \\
\text { (b) }\end{array}$} & $\begin{array}{l}\text { Revenus } \\
\text { immob. }\end{array}$ & Actifs & Autres & Total & $\begin{array}{c}\text { nb. } \\
\text { meneges }\end{array}$ \\
\hline Paurres & 20.949 & 26.205 & 17.693 & 20.487 & 2.371 & 15.83 & 19.534 & 29.5 \\
Non pauvres & 79.051 & 73.795 & 82.307 & 79.513 & 97.629 & 84.17 & 80.466 & 70.5 \\
Ties catég. & 100 & 100 & 100 & 100 & 100 & 100 & 100 & 100 \\
Très pauvr. & 2.103 & 3.346 & 2.462 & 3.048 & 0.002 & 1.90 & 2.241 & 4.5 \\
Autres & 97.897 & 69.654 & 97.538 & 96.952 & 99.998 & 98.10 & 97.759 & 95.5 \\
\hline
\end{tabular}

Tableau A20 : Source de revenus des ménages urbains par groupes de pauvreté (1990/91)

\begin{tabular}{|l|c|c|c|c|c|c|c|}
\hline \multicolumn{1}{|c|}{ Groupes } & Salaires & $\begin{array}{c}1 \\
\text { (a) }\end{array}$ & $\begin{array}{c}1 \\
\text { (b) }\end{array}$ & $\begin{array}{c}\text { Revenus } \\
\text { mmmob. }\end{array}$ & Actifs & Autres & Total \\
\hline Pauvres & 56.853 & 7.142 & 20.245 & 5.854 & 0.215 & 9.692 & 100 \\
Non paurres & 52.081 & 4.883 & 22.864 & 5.516 & 2.147 & 12.510 & 100 \\
Ttes catég. & 53.013 & 5.324 & 22.352 & 5.582 & 1.769 & 11.960 & 100 \\
Très pauvr. & 49.753 & 7.949 & 24.555 & 7.591 & 0.002 & 10.150 & 100 \\
Autres & 53.065 & 5.283 & 22.317 & 5.550 & 1.797 & 11.988 & 100 \\
\hline
\end{tabular}

Tableau A21 : Origine des revenus des ménages ruraux par groupes de pauvreté (1990/91)

\begin{tabular}{|l|r|r|r|r|r|r|r|r|}
\hline Groupes & Salaires & \multicolumn{1}{c|}{$\begin{array}{c}1 \\
\text { (a) }\end{array}$} & \multicolumn{1}{c|}{$\begin{array}{c}1 \\
\text { (b) }\end{array}$} & $\begin{array}{l}\text { Fevenus } \\
\text { immob. }\end{array}$ & Actíls & Autres & \multicolumn{1}{c|}{ Tolal } & $\begin{array}{c}\text { nb. } \\
\text { ménages }\end{array}$ \\
\hline Pauvres & 20.857 & 14.376 & 13.158 & 13.370 & 6.094 & 14.30 & 15.838 & 20.9 \\
Non pauvres & 79.143 & 85.624 & 86.842 & 86.630 & 93.906 & 85.70 & 84.162 & 79.1 \\
Ties catég. & 100 & 100 & 100 & 100 & 100 & 100 & 100 & 100 \\
Très pauvr. & 3.316 & 0.956 & 1.763 & 1.588 & 0.000 & 5.155 & 1.954 & 4.9 \\
Autres & 96.684 & 99.044 & 98.237 & 98.412 & 100 & 94.85 & 98.046 & 95.1 \\
\hline
\end{tabular}

Table A22 : Source de revenus des ménages ruraux par groupes de pauvreté (1990/91)

\begin{tabular}{|l|c|c|c|c|c|c|c|}
\hline \multicolumn{1}{|c|}{ Groupes } & Salaires & $\begin{array}{c}1 \\
\text { (a) }\end{array}$ & $\begin{array}{c}1 \\
\text { (b) }\end{array}$ & $\begin{array}{c}\text { Revenus } \\
\text { immob. }\end{array}$ & Actils. & Aulres & Total \\
\hline Pauvres & 34.853 & 44.419 & 10.076 & 5.301 & 0.177 & 5.173 & 100 \\
Non pauvres & 24.887 & 49.785 & 12.515 & 6.464 & 0.514 & 5.835 & 100 \\
Ttes calég. & 26.465 & 48.935 & 12.129 & 6.280 & 0.461 & 5.730 & 100 \\
Très pauvres & 44.907 & 23.932 & 10.941 & 5.104 & 0.000 & 15.117 & 100 \\
Autres & 26.401 & 49.023 & 12.130 & 6.284 & 0.463 & 5.697 & 100 \\
\hline
\end{tabular}

Source : Résultats préliminaires de l'enquête sur les revenus et dépenses 1990/91, CAPMAS, 1992.

1 (a) revenus du travail non salarié agricole.

1 (b) revenus du travail non salarié non agricole. 
Tableau A23 : Indices de pauvreté des ménages urbains (par occupation) en 1981/82 (seuil de pauvreté $=960.845$ LE par ménage)

\begin{tabular}{|c|c|c|c|c|c|c|c|}
\hline \multirow{2}{*}{ Occupation } & \multicolumn{3}{|c|}{ Indices de pauvreté } & \multicolumn{3}{|c|}{ Contribution } & \multirow{2}{*}{$\begin{array}{c}\text { nb. } \\
\text { merages }\end{array}$} \\
\hline & PO & P1 & P2 & $P O$ & P1 & $P 2$ & \\
\hline Indépendants & 23.38 & 8.03 & 4.50 & 8.35 & 10.14 & 12.03 & 12.111 \\
\hline Administr. & 6.38 & 1.01 & 0.05 & 0.58 & 0.32 & 0.03 & 3.082 \\
\hline Employes & 24.32 & 3.79 & 1.29 & 6.03 & 3.32 & 2.40 & 8.405 \\
\hline Commenco & 31.01 & 8.39 & 2.47 & 8.48 & 8.10 & 5.05 & 9.269 \\
\hline Services & 42.38 & 11.68 & 5.23 & 12.99 & 12.65 & 11.99 & 10.395 \\
\hline $\begin{array}{l}\text { Travailleurs } \\
\text { agnicoles }\end{array}$ & 43.01 & 10.57 & 4.30 & 10.51 & 9.13 & 7.85 & 8.285 \\
\hline $\begin{array}{l}\text { Travailleurs } \\
\text { de lindustrie }\end{array}$ & 29.99 & 6.05 & 2.44 & 24.20 & 12.24 & 14.73 & 27.369 \\
\hline $\begin{array}{l}\text { Non } \\
\text { actifs }\end{array}$ & 46.45 & 17.79 & 9.87 & 28.88 & 39.10 & 45.91 & 21.084 \\
\hline Total & 33.86 & 9.58 & 4.53 & 100 & 100 & 100 & 100 \\
\hline
\end{tabular}

Tableau A24 : Indices de pauvreté des ménages ruraux (par occupation) en 1981/82, (seuil de pauvreté $=960,845$ par ménage)

\begin{tabular}{|c|c|c|c|c|c|c|c|}
\hline \multirow{2}{*}{ Oxcipation } & \multicolumn{3}{|c|}{ Indices de paurrete } & \multicolumn{3}{|c|}{ Contribution } & \multirow{2}{*}{$\begin{array}{c}\text { nib de } \\
\text { menages }(\%)\end{array}$} \\
\hline & PD & $P 1$ & $P 2$ & PO & P1 & P2 & \\
\hline Indépendants & 41.22 & 18.64 & 12.07 & 9.23 & $12.7 t$ & 16.13 & 6.184 \\
\hline Administr. & 2.50 & 0.26 & 0.03 & 0.04 & 0.01 & 0.003 & 0.488 \\
\hline Employes & 10.43 & 2.03 & 0.80 & 0.75 & 0.44 & 0.35 & 1.987 \\
\hline commence & 16.81 & 4.51 & 1.96 & 2.65 & 2.17 & 1.84 & 4.352 \\
\hline Services & 21.54 & 5.57 & 2.50 & 5.30 & 4.18 & 3.68 & 6.802 \\
\hline $\begin{array}{l}\text { Travailleurs } \\
\text { agricoles }\end{array}$ & 22.42 & 5.39 & 2.10 & 42.03 & 30.77 & 23.51 & 51.762 \\
\hline $\begin{array}{l}\text { Travailleurs de } \\
\text { Pindustrie }\end{array}$ & 16.00 & 4.03 & 1.62 & 6.36 & 4.88 & 3.84 & 10.972 \\
\hline $\begin{array}{l}\text { Non } \\
\text { actits }\end{array}$ & 53.21 & 23.27 & 13.44 & 33.64 & 44.83 & 50.67 & 17.457 \\
\hline Total & 27.61 & 9.06 & 4.63 & 100 & 100 & 100 & 100 \\
\hline
\end{tabular}

Source : enquête 1981/82 sur le budget des familles, CAPMAS, 1992. 
Tableau A25: Indices de pauvreté des ménages urbains par activité en 1981/82, (seuil de pauvreté $=960345$ LE par ménage)

\begin{tabular}{|c|c|c|c|c|c|c|c|}
\hline \multirow{2}{*}{ Activite } & \multicolumn{3}{|c|}{ Indices de pawreté } & \multicolumn{3}{|c|}{ Controbution } & \multirow{2}{*}{$\begin{array}{c}\text { nb. } \\
\text { merenges }\end{array}$} \\
\hline & PO & $P_{1}$ & P2 & PO & P1 & $P 2$ & \\
\hline Agricullure & 40.90 & 10.08 & 4.21 & 14.80 & 15.13 & 14.23 & 11.110 \\
\hline Ind minięre & 4,17 & 0.48 & 0.06 & 0.05 & 0.02 & 0.01 & 0.332 \\
\hline Industrie & 30.50 & 6.14 & 2.27 & 21.18 & 17.60 & 14,65 & 21208 \\
\hline Electricité & 25.00 & 4.35 & 1.28 & 0.93 & 0.67 & 0.44 & 1.136 \\
\hline Construction & 25.71 & 4.41 & 1.37 & 5.31 & 3.75 & 2.63 & 6.303 \\
\hline Commerce & 30.51 & 8.00 & 3.71 & 15.88 & 17.19 & 17.99 & 15.889 \\
\hline Transports & 23.95 & 4.70 & 2.08 & 7.49 & 6.07 & 6.04 & 9.554 \\
\hline Finances & 12.50 & 2.53 & 0.73 & 0.82 & 0.68 & 0.44 & 1.995 \\
\hline Services & 28.51 & 6.82 & 3.13 & 28.81 & 26.46 & 27.34 & 29.716 \\
\hline Autres & 54.04 & 24.41 & 14.16 & 6.67 & 12.43 & 16.24 & 3.768 \\
\hline Total & 30.54 & 7.40 & 3.28 & 100 & 100 & 100 & 100 \\
\hline
\end{tabular}

Source : enquête sur les budgets des familles 1981/82, CAPMAS, 1992

Tableau A26: Indices de pauvreté des ménages ruraux par activité en 1981/82, (seuil de pauvreté $=960845$ LE par ménage)

\begin{tabular}{|c|c|c|c|c|c|c|c|}
\hline \multirow{2}{*}{ Aclivite } & \multicolumn{3}{|c|}{ Indices de pauvieté } & \multicolumn{3}{|c|}{ Contribution } & \multirow{2}{*}{$\begin{array}{c}\text { nb. } \\
\text { menags }\end{array}$} \\
\hline & PO & P1 & $P 2$ & $\mathrm{PO}$ & P1 & $\overline{\text { P2 }}$ & \\
\hline Agriculture & 22.31 & 5.32 & 2.86 & 66.64 & 57.33 & 55.84 & 63.231 \\
\hline Ind minière & 9.09 & 4.71 & 2.44 & 0.07 & 0.14 & 0.12 & 0.162 \\
\hline Industria & 16.92 & 4.59 & 1.82 & 6.21 & 6.29 & 4.36 & 7.767 \\
\hline Electriciłe & 5.26 & 1.28 & 0.31 & 0.07 & 0.06 & 0.03 & 0.281 \\
\hline Construction & 11.11 & 2.70 & 1.24 & 1.44 & 1.31 & 1.06 & 2.747 \\
\hline Commerce & 17.27 & 4.64 & 2.05 & 5.30 & 5.32 & 4.11 & 6.497 \\
\hline Transports & 14.10 & 4.79 & 2.78 & 1.54 & 1.95 & 1.97 & 2.304 \\
\hline Finarces & 5.56 & 0.58 & 0.06 & 0.14 & 0.06 & 0.01 & 0.532 \\
\hline Services & 19.06 & 4.88 & 2.12 & 11.09 & 10.60 & 8.04 & 12.315 \\
\hline Autres & 63.77 & 30.64 & 19.04 & 12.55 & 22.51 & 24,46 & 4.164 \\
\hline Total & 21.16 & 5.67 & 3.24 & 100 & 100 & 100 & 100 \\
\hline
\end{tabular}

Source : enquête sur les budgets des familles 1981/82, CAPMAS, 1992. 
Tableau A27: Indices de pauvreté des ménages urbains par niveau d'éducation en 1981/82

\begin{tabular}{|c|c|c|c|c|c|c|c|}
\hline \multirow{2}{*}{$\begin{array}{c}\text { Niveaul } \\
\text { féducation }\end{array}$} & \multicolumn{3}{|c|}{ Indices de pauvrelé } & \multicolumn{3}{|c|}{ Contsbution } & \multirow{2}{*}{$\begin{array}{c}\text { nb. } \\
\text { menages }\end{array}$} \\
\hline & PO & P1 & P2 & Po & P1 & P2 & \\
\hline Anaíphabète & 41.38 & 15.90 & 9.12 & 0.92 & 1.23 & 1.37 & 0.640 \\
\hline $\begin{array}{l}\text { Enseignement } \\
\text { primatre }\end{array}$ & 40.61 & 12.02 & 5.98 & 64.13 & 88.59 & 89.40 & 70.351 \\
\hline $\begin{array}{l}\text { Enseignement } \\
\text { secondaire }\end{array}$ & 22.75 & 4.25 & 1.76 & 11.70 & 7.71 & 6.55 & 17.460 \\
\hline $\begin{array}{l}\text { Enseignement } \\
\text { universitaire }\end{array}$ & 15.24 & 4,34 & 2.22 & 1.03 & 1.03 & 1.08 & 2.292 \\
\hline $\begin{array}{l}\text { Enseignement } \\
\text { post-univers. }\end{array}$ & 8.22 & 1.67 & 1.01 & 2.22 & 1.59 & 1.96 & 9.155 \\
\hline Tolal & 33.96 & 9.62 & 4.71 & 100 & 100 & 100 & 100 \\
\hline
\end{tabular}

Source : enquête sur les budgets des familles 1981/82, CAPMAS, 1992.

Tableau A28: Indices de pauvreté des ménages ruraux par niveau d'éducation en 1981/82. Source: enquête sur les budgets des familles 1981/82, CAPMAS, 1992

\begin{tabular}{|c|c|c|c|c|c|c|c|}
\hline \multirow{2}{*}{$\begin{array}{c}\text { Niveau } \\
\text { Jéducation }\end{array}$} & \multicolumn{3}{|c|}{ Indices de pauvreté } & \multicolumn{3}{|c|}{ Contrbution } & \multirow{2}{*}{ ménages. } \\
\hline & PO & 81 & P2 & Po. & $P 1$ & P2 & \\
\hline Analphabete & 51.15 & 25.23 & 16.98 & 1.18 & 1.87 & 2.12 & 0.572 \\
\hline $\begin{array}{l}\text { Enseignement } \\
\text { primaire }\end{array}$ & 28.70 & 9.29 & 4.68 & 96.26 & 95.76 & 95.40 & 93.416 \\
\hline $\begin{array}{l}\text { Enseignement } \\
\text { secondaire }\end{array}$ & 15.38 & 4,14 & 1.89 & 2.47 & 2.02 & 1,83 & 4.430 \\
\hline $\begin{array}{l}\text { Enseignement } \\
\text { universitaire }\end{array}$ & 6.07 & 1.62 & 0.44 & 0.22 & 0.16 & 0.09 & 0.913 \\
\hline $\begin{array}{l}\text { Enseignement } \\
\text { post-univers. }\end{array}$ & 7.27 & 2.52 & 1.17 & 0.18 & 0.19 & 0.17 & 0.669 \\
\hline Tolal & 27.63 & 9.06 & 4.57 & 100 & 100 & 100 & 100 \\
\hline
\end{tabular}

\section{NOTES}

1. ATKINSON A.B., « On the measurement of poverty », Econometrica, 55, 1987.

2. SEN A.K., The standard of living, Cambridge, Cambridge University Press, 1987.

3. RAVALLION M., "Poverty comparisons - A guide to concepts and methods ", LSMS Working paper $\mathrm{n}^{\circ}$ 88, World Bank, Washington D.C., 1992, p. 35.

4. FOSTER J., GREER J. et THORBECKE, "A class of decomposable poverty measures ", Econometrica, vol. 52, 1984, p. 761-765. 
5. KANBUR R., "The implication of adjustment programs for poverty: conceptual issues and analytical framework». Paper presented to IMF, 1988.

6. KORAYEM K., "The impact of economic adjustment policies on the vulnerable families and children in Egypt », The Third world Forum and UNICEF, 1987.

7. IBRAHIM S., " Social mobility and income distribution in Egypt », 1952-1977. 'Abd al-Khalik G. and Tignor R.eds, The political-economy of income distribution in Egypt, Holmes and Meier publishers, London, 1982.

8. Cf. KORAYEM, op. cit., p. 127-129.

9. BADRAN A., "Structural adjustments in health sector to protect vulnerable groups ». The Third World Forum and UNICEF, 1989.

10. Prévisions du FMI, République arabe d'Égypte, Staff Report for 1992 Article IV Consultations, p. 6, août 1992.

11. ARE current economic situation and medium term prospects, World Bank Report, Egypt Division, EMENA Region, mars 1985, tableau 5.1 de l'annexe statistique.

12. BESLEY T.J., KANBUR R., « Food subsidies and poverty alleviation », Economic Journal, 1988.

13. Il faut relever que K. KORAYEM a évalué, en 1987, les seuils de pauvreté pour l'année 1981/82 à 944,0 LE et 704,0 LE pour les ménages urbains et ruraux respectivement.

\section{INDEX}

Mots-clés : économie, pauvreté

\section{AUTEURS}

HEBA AL-LAITHY

Université du Caire

HANAA KHEIR AL-DIN

Université du Caire 